\title{
Continuation of Periodic Solutions in the Waveguide Array Mode-Locked Laser
}

\author{
Matthew O. Williams ${ }^{\mathrm{a}, *}$, Jon Wilkening ${ }^{\mathrm{b}}$, Eli Shlizerman ${ }^{\mathrm{a}}$, J. Nathan Kutz ${ }^{\mathrm{a}}$ \\ ${ }^{a}$ Department of Applied Mathematics, University of Washington, Seattle, WA, 98195-2420 \\ ${ }^{b}$ Department of Mathematics, University of California, Berkeley, CA 94720-3840
}

\begin{abstract}
We apply the adjoint continuation method to construct highly-accurate, periodic solutions that are observed to play a critical role in the multi-pulsing transition of mode-locked laser cavities. The method allows for the construction of solution branches and the identification of their bifurcation structure. Supplementing the adjoint continuation method with a computation of the Floquet multipliers allows for explicit determination of the stability of each branch. This method reveals that, when gain is increased, the multi-pulsing transition starts with a Hopf bifurcation, followed by a period-doubling bifurcation, and a saddle-node bifurcation for limit cycles. Finally, the system exhibits chaotic dynamics and transitions to the double-pulse solutions. Although this method is applied specifically to the waveguide array mode-locking model, the multi-pulsing transition is conjectured to be ubiquitous and these results agree with experimental and computational results from other models.
\end{abstract}

Keywords:

\section{Introduction}

High-power pulsed lasers are an increasingly important technological innovation. Their conjectured and envisioned applications, ranging from military devices and precision medical surgery to optical interconnection networks [1], have grown significantly over the past two decades. Such lasers are one of the few examples of a commercially viable photonics technology that are based fundamentally on nonlinear processes. As a result, mode-locking technologies have placed

\footnotetext{
${ }^{*}$ Corresponding author

Email address: mowill@amath. washington.edu (Matthew O. Williams) Preprint submitted to Physica D
}

June 22, 2011 

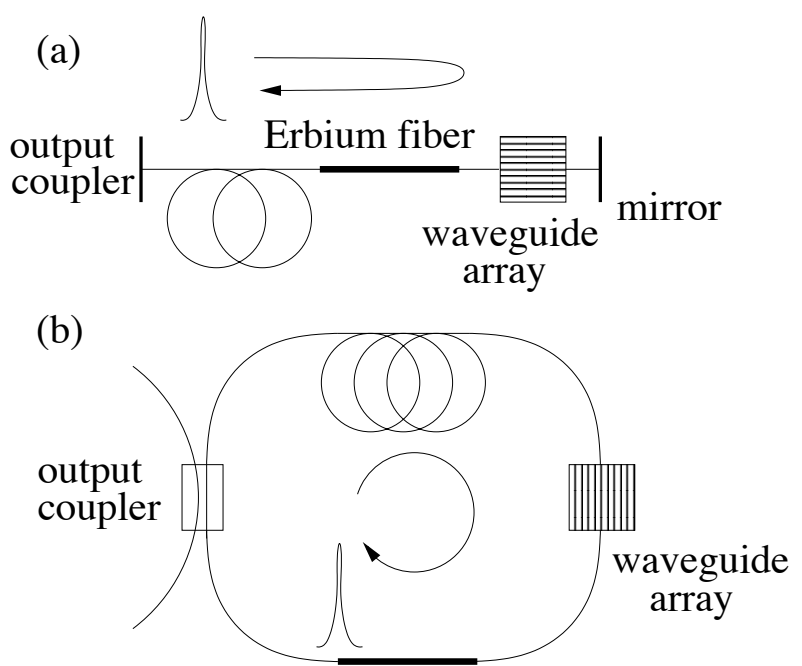

Erbium fiber

Figure 1: Two possible laser cavity configurations that include nonlinear mode-coupling from the waveguide array as the mode-locking element. The fiber coupling in and out of the waveguide array occurs at the central waveguide as illustrated. Any electromagnetic field that is propagated into the neighboring waveguides is ejected (attenuated) from the laser cavity. In addition to the basic setup, polarization controllers, isolators, and other stabilization mechanisms may be useful or required for successful operation.

a premium on the engineering and optimization of laser cavities that are aimed at producing output pulses of tens to hundreds of femtoseconds with maximal peak powers in the kilowatt range and energies exceeding 10 nanojoules. Such technological demand has pushed mode-locked lasers to the forefront of commercially viable, nonlinear photonic devices. One of the most recently envisioned methods for generating stable mode-locking incorporates the intensity discrimination induced by the nonlinear mode-coupling properties in a waveguide array [2-6]. The waveguide array mode-locking produces robust mode-locking and displays the ubiquitous multi-pulsing transition instability [7,8] whereby an increase in the laser cavity energy above a given threshold causes a single pulse per round trip to bifurcate to two pulses per round trip. This multi-pulsing transition dynamics is the primary focus of this manuscript.

Figure 1 illustrates two possible mode-locking configurations in which the waveguide array provides the critical effect of intensity discrimination (saturable absorption) [1,9]. In Fig. 1(a) a linear cavity configuration is considered whereas in Fig. 1(b) a ring cavity geometry is considered. In either case, the waveguide array provides an intensity dependent pulse shaping by coupling out low intensity wings to the neighboring waveguides through a process called nonlinear mode- 
coupling.

Optical nonlinear mode-coupling (NLMC) is a well-established phenomenon that has been both experimentally verified [10-14] and theoretically characterized [15-17]. NLMC has been an area of active research in all-optical switching and signal processing applications using waveguide arrays [11-14], dual-core fibers $[10,15,16]$, and fiber arrays $[18,19]$. It is only recently that the temporal pulse shaping associated with NLMC has been theoretically proposed for the passive intensity-discrimination element in a mode-locked fiber laser [2,3]. The models derived to characterize the mode-locking consist of two governing equations: one for the fiber cavity and a second for the NLMC element $[2,3]$ (See Fig. 1). Although the two discrete components provide accurate physical models for the laser cavity, characterizing the underlying laser stability and dynamics is often analytically intractable. Thus, it is helpful to construct an averaged approximation to the discrete components model in order to approximate and better understand the mode-locking behavior. Indeed, the essence of Haus' master mode-locking theory [1] is approximating discrete elements with a continuous model. The same approach is used here to generate a continuous system of governing equations from a system that would, due to the inclusion of the waveguide array and Erbium fiber, include discrete effects $[4,5]$.

Even with these continuous models, such as the waveguide array modelocking model (WGAML) [2-5] used in this manuscript, an accurate characterization of the bifurcation structure had not been performed. Specifically, little was known about the branch of observed $z$-periodic breather solutions. What work has been done involves approximating the bifurcation sequence qualitatively using principal components in a low-dimensional reduction [6].

In this work, we use a hybrid numerical method, called the adjoint continuation method (ACM), that is able to calculate arbitrarily accurate solutions and perform a PDE bifurcation study. In particular, the method reveals the key aspects about and the complexity of the bifurcation structure of the multi-pulsing instability, an overview of which is shown in Figure 2. The branches of solutions believed to be involved in the multi-pulsing transition can be separated into four qualitatively different types: stationary one-pulse (single-pulse) solutions, period-one breather solutions, period-two breather solutions, and stationary twopulse (double-pulse) solutions with an example of each shown in the bottom of Figure 2. The stationary one- and two- pulse solutions posses a constant amplitude but a linearly increasing phase. The period-one breather solutions have a $z$ periodic amplitude but are even functions over the entire period up to a translation in $t$. The period-two breather solutions are also $z$ periodic in amplitude, but 


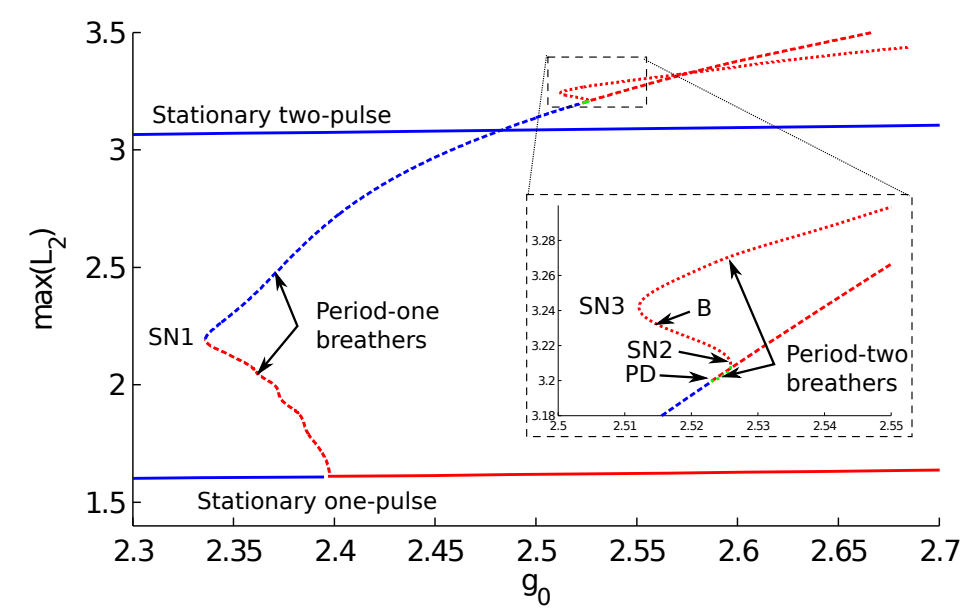

Stationary one-pulse Period-one breather Period-two breather Stationary two-pulse

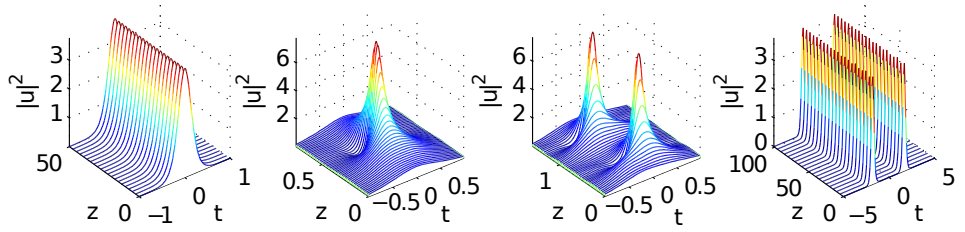

Figure 2: (Color online, two columns) (Top) Bifurcation diagram including the branches of stationary one-pulse, stationary two-pulse, period-one breather, and period-two breather solutions. Branches in blue or green are linearly stable while branches in red are linearly unstable. Branches in solid lines are from stationary (constant amplitude) solutions while branches in dashed lines are $z$-periodic solutions. The green dashed lines represent period-two breathers and the blue lines period-one. Hopf, saddle-node, and period-doubling bifurcations are denoted by $H, S N$, and $P D$ respectively. A fourth unknown bifurcation is indicated by $B$. (Bottom) Examples of the four qualitatively different solution behaviors - stationary one-pulse (single-pulse) solutions, period-one breathers, period-two breathers, and stationary two-pulse (double-pulse) solutions observed during the multi-pulsing transition. The stationary two-pulse solutions can be treated as two non-interacting stationary one-pulse solutions.

they are neither even nor odd functions for the entire period.

By studying the stability of solution branches, we find that a subcritical Hopf bifurcation occurs on the one pulse solution branch, at the point labeled $\mathrm{H}$ in Figure 2. This generates a branch of period-one breather solutions. This period-one (breather) branch first undergoes a saddle-node bifurcation (SN1) which is followed by a period-doubling bifurcation (PD). This period-doubling bifurcation creates the branch of period-two breather solutions. This period-two solution branch also undergoes three bifurcations, labeled SN2, B, and SN3, and eventually leads to complex spatio-temporal (chaotic-like) behavior. Coexisting with 
these solution branches is a stationary two-pulse solution branch. This bifurcation diagram represents the underlying nonlinear phenomenon of the multipulsing transition dynamics. A detailed account of each solution branch and its complex transitions is developed herein. These results extend and justify the results of previous qualitative efforts in [6] and also have revealed new information about the source of symmetry breaking. Further our results hint at the mechanism for the onset of spatial-temporal disorder in the WGAML. Furthermore, they are also consistent with recent experimental observations of the transition dynamics in laser cavities [20,21].

The paper is arranged as follows: Sec. 2 gives a brief overview of the governing averaged equations in the laser cavity. Section 3 develops the algorithm necessary for computing solution branches and following bifurcations to new paths of solutions. The bifurcation structure of the waveguide array mode-locked laser is given in Sec. 4. A brief summary and outlook for the method and the laser system is given in Sec. 5 .

\section{Governing Equations}

When placed within an optical fiber cavity, the pulse shaping mechanism of the waveguide array leads to stable and robust mode-locking [2, 3]. In its simplest form, the nonlinear mode-coupling is averaged into the laser cavity dynamics [5]. Numerical simulations have shown that the fundamental behavior in the laser cavity does not change when considering more than five waveguides [5]. Further simplifications to the five waveguide model can be achieved by making use of the symmetric nature of the coupling and lower intensities in the neighboring waveguides [4]. The resulting approximate evolution dynamics describing the waveguide array mode-locking model (WGAML) is given by

$$
\begin{aligned}
& i \frac{\partial u}{\partial z}+\frac{D}{2} \frac{\partial^{2} u}{\partial t^{2}}+\beta|u|^{2} u+C v+i \gamma_{0} u-i g(z)\left(1+\tau \frac{\partial^{2}}{\partial t^{2}}\right) u=0 \\
& i \frac{\partial v}{\partial z}+C(w+u)+i \gamma_{1} v=0 \\
& i \frac{\partial w}{\partial z}+C v+i \gamma_{2} w=0
\end{aligned}
$$

with

$$
g=\frac{2 g_{0}}{1+\|u\|^{2} / e_{0}} .
$$

Here $u(z, t)$ is the electric field in the 0th waveguide while the $v(z, t)$ and $w(z, t)$ fields model the electromagnetic energy in the neighboring channels of the waveguide array. Note that the equations are posed on the infinite domain so that 

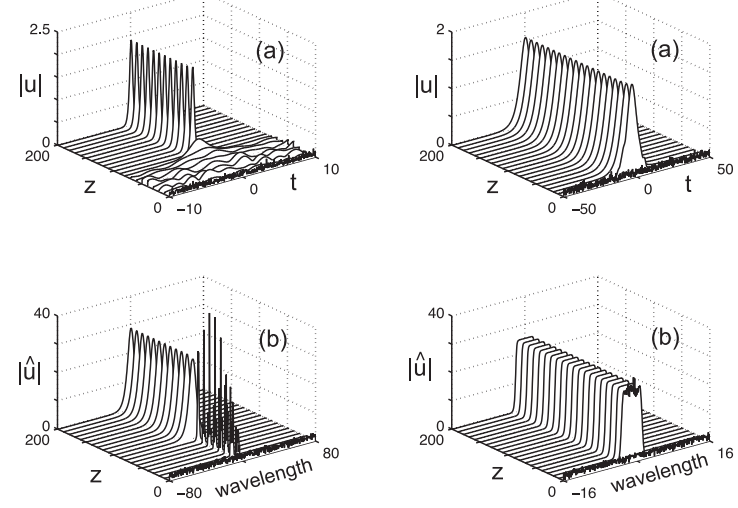

Figure 3: Typical (a) time and (b) spectral mode-locking dynamics of the waveguide array modelocking model Eq. (1) in the anomalous (left) and normal (right) dispersion regime from initial white-noise. For anomalous dispersion, the steady state solution is a short, nearly transformlimited pulse that acts as an attractor to the mode-locked system. For normal dispersion, the steady state solution is a broad, highly-chirped pulse that acts as an attractor to the mode-locked system.

$u, v, w \rightarrow 0$ as $x \rightarrow \pm \infty$. Further, since the gain $g(t)$ saturates with the $L_{2}$ norm of the field, spatially $(t)$ periodic solutions (e.g. wavetrains) are not allowed due to physical considerations, i.e. they would have infinite energy. The equations governing the neighboring fields are ordinary differential equations. All fiber propagation and gain effects occur in the central waveguide. It is this approximate system that will be the basis for our numerical study. Additionally, the simplified system in (1) provides a great deal of analytic insight due to its hyperbolic secant solutions

$$
u(z, t)=\eta \operatorname{sech}(\omega t)^{1+i A} e^{i \theta z},
$$

where the solution amplitude $\eta$, width $\omega$, chirp parameter $A$, and phase $\theta$ satisfy a set of nonlinear equations [4]. This limiting solution forms from low amplitude white-noise initial conditions and appears to have a large basin of attraction. This is in contrast to the master mode-locked equation [1] for which initial conditions must be carefully prepared to observe stable mode-locking.

In the anomalous dispersion regime $(D=1>0)$, soliton-like pulses can be formed as a result of the balance of anomalous dispersion and positive (i.e. selffocusing) nonlinearity. Typically, mode-locked fiber lasers operating in the anomalous dispersion regime are limited in pulse energy by restrictions among the soliton parameters; this is often referred to as the soliton area theorem. However, 
ultra-short, nearly transform-limited output pulses are desired for many applications. This encourages exploration of possible laser cavity configurations that could potentially maximize pulse energy in the anomalous dispersion regime. Figure 3 (left panel) shows the typical time- and spectral-domain mode-locking dynamics of the waveguide array model (1) in the anomalous dispersion regime. The equation parameters used throughout the text are:

$$
\left(\beta, C, \gamma_{0}, \gamma_{1}, \gamma_{2}, e_{0}\right)=(8,5,0,0,10,1) \text {. }
$$

The gain, $g_{0}$, will be altered throughout the text, but is 1.5 for the purposes of Figure 3. Stable and robust mode-locking is achieved from initial white-noise after $z \sim 100$ units. The steady state pulse solution has a short pulse duration and is nearly transform-limited, which is in agreement with experiments performed in the anomalous dispersion regime [1].

Mode-locking in the normal dispersion regime $(D=-1<0)$ relies on non-soliton processes and has been shown experimentally to have stable highchirped, high-energy pulse solutions. Figure 3 (right panel) shows the typical time and spectral mode-locking dynamics of the waveguide array model (1) in the normal dispersion regime. Here the equation parameters are $\beta=1, C=3$, $\gamma_{0}=0, \gamma_{1}=1, \gamma_{2}=10, g_{0}=10$, and $e_{0}=1$. In contrast to mode-locking in the anomalous dispersion regime, the mode-locked solution is quickly formed from initial white-noise after $z \sim 10$ units. The mode-locked pulse is broad in the time domain and has the squared-off spectral profile characteristic of a highly chirped pulse $(A \gg 1)$. These characteristics are in agreement with observed experimental pulse solutions in the normal dispersion regime. Although the large pulse-width and high chirp make the pulse solutions impractical for many photonic applications, the potential for high-energy pulses from normal dispersion mode-locked lasers has generated a great deal of interest.

\section{The Adjoint Continuation Method (ACM)}

In this section we describe the ACM for tracking families of time-periodic (or in this case, $z$-periodic) solutions by numerical continuation. Starting with mode-locked solutions of the form of Eq. (3), which are trivially z-periodic (up to a phase), we will use the method to follow these paths through a sequence of bifurcations leading to more and more complicated, ultimately chaotic, dynamics. Tracking chaotic solutions is beyond the capabilities of the ACM, but it has been observed by Kutz and Standstede [4] that the chaotic solutions, upon further increase of the gain, lead to the formation of the double-pulse solutions. This bifurcation sequence is described in Section 4. 
General methods [22-25] for the solution of nonlinear two-point boundary value problems tend to be geared toward ordinary differential equations, and can be prohibitively expensive for partial differential equations. Recently [2628], Wilkening and Ambrose introduced an efficient method of computing timeperiodic solutions of nonlinear PDEs. We will refer to this method as the Adjoint Continuation Method (ACM). The idea is to develop a variant of a shooting method $[29,30]$ in which a nonlinear functional of the initial condition is minimized using adjoint-based optimal control methods [31-33] to obtain a solution of the boundary value problem. Key challenges in adapting this method to the system in Eq. (1) include finding solutions that are only periodic up to a phase; incorporating the gain, $g_{0}$, in the adjoint system to allow other variables (such as the period) to be used as bifurcation parameters; and adapting high order, semi-implicit Runge-Kutta methods $[34,35]$ to handle the case when the terms responsible for stiffness (those involving $\partial^{2} u / \partial t^{2}$ in (1)) depend non-linearly on $u$ through a gain $g$ that depends on $\|u\|$.

Recall that $z$ is the time-like variable in (1) while $t$ is the space-like variable. To facilitate the use of spectral methods, we adopt periodic boundary conditions over an interval $t \in[-L, L)$ where $L=20 . L$ is chosen large enough that the solution, which decays exponentially, is of order $10^{-10}$ near $t= \pm L$. Once $L$ is large enough, the solution is insensitive to further changes in $L$, and may be regarded as a solution over $\mathbb{R}$ without periodic boundary conditions. In what follows, we take $L=\pi$ and absorb the appropriate factors from the change of variables into $D, \tau$ and $e_{0}$ in (1) and (2). However, for the plots in Section 4, we transform back to the original domain.

Following the basic approach in [26-28], we define a nonlinear functional $G$ of the initial conditions and supposed period that is zero if and only if the solution is $z$-periodic. As we wish to determine the dependence of $G$ on the parameter $g_{0}$ in (2), we add to (1) the equation $\partial g_{0} / \partial z=0$. This will be explained in more detail below. We also wish to find solutions that are only z-periodic up to a phase. An easy way to do this is to include extra terms on the right hand side of (1a)(1c) that affect the solution by multiplying $u(z, t), v(z, t)$ and $w(z, t)$ by $e^{-i \theta z}$; we then search for $\theta$ such that the new problem has fully $z$-periodic solutions. The 
new set of equations is

$$
\begin{aligned}
i \frac{\partial u}{\partial z}+\frac{D}{2} \frac{\partial^{2} u}{\partial t^{2}}+\beta|u|^{2} u+C v+i \gamma_{0} u-\frac{2 i g_{0}}{1+\|u\|^{2} / e_{0}}\left(1+\tau \frac{\partial^{2}}{\partial t^{2}}\right) u & =\theta u, \\
i \frac{\partial v}{\partial z}+C(w+u)+i \gamma_{1} v & =\theta v, \\
i \frac{\partial w}{\partial z}+C v+i \gamma_{2} w & =\theta w \\
\frac{\partial g_{0}}{\partial z} & =0 .
\end{aligned}
$$

Although $u, v$ and $w$ in (1) are complex valued, we represent the state of the system (with $z$ frozen) as an element of the real Hilbert space

$$
X=\left\{\left(u, v, w, g_{0}\right): u, v, w \in L^{2}((-\pi, \pi], \mathbb{C}), g_{0} \in \mathbb{R}\right\}
$$

with inner product

$$
\left\langle q_{1}, q_{2}\right\rangle=\int_{-\pi}^{\pi} \mathfrak{R}\left\{u_{1}(t)^{*} u_{2}(t)+v_{1}(t)^{*} v_{2}(t)+w_{1}(t)^{*} w_{2}(t)\right\} d t+g_{0,1} g_{0,2}
$$

where, $q_{i}(t)=\left(u_{i}(t), v_{i}(t), w_{i}(t), g_{0, i}\right) \in X$. Next we define

$$
G\left(q_{0}, Z, \theta\right)=\frac{1}{2}\left\|q(Z, \cdot)-q_{0}(\cdot)\right\|^{2}, \quad\|q\|^{2}=\langle q, q\rangle,
$$

where $q(z, t)$ solves the initial value problem (1d)-(1g) with initial conditions $q(0, t)=q_{0}(t)$. We note that $G\left(q_{0}, Z, \theta\right)=0$ if and only if $q(z, t)$ is $z$-periodic, with period $Z$.

To evaluate $G$ numerically, we evolve $q(z, t)$ using a spectral collocation method in $t$ and a 5th order semi-implicit Runge-Kutta method in $z$, that is described in detail in Appendix C. A prescribed fraction of the Fourier modes (around 40\%) are allowed to be non-zero in $q_{0}(t)$. The remaining (high frequency) modes of the initial condition are set to zero to avoid aliasing errors in the computation of $G$. To minimize $G$, we use the Broyden-Fletcher-GoldfarbShanno (BFGS) algorithm [36] to vary $Z, \theta, g_{0}$ and the non-zero Fourier modes of the initial condition. One of these variables (usually $g_{0}$ or $Z$ ) is taken as a bifurcation parameter in the continuation algorithm and removed from the list of variables that BFGS is allowed to vary in search of a minimum. Alternatively, a penalty function [26] can be used to enforce the value of the bifurcation parameter. In either approach, it is useful to be able to use any variable as a bifurcation 
parameter and switch between them as necessary to traverse turning points and avoid ill-conditioned minimization problems.

BFGS is a quasi-Newton gradient descent method that builds up an approximate inverse Hessian matrix based on the sequence of gradient vectors it encounters in the course of the line searches. Thus, to use the algorithm, we must be able to accurately and efficiently compute the gradient, $\nabla G$. Some of the components of $\nabla G$ can be computed immediately, e.g.

$$
\frac{\partial G}{\partial Z}=\left\langle\frac{\partial q}{\partial z}(Z, \cdot), q(Z, \cdot)-q_{0}(\cdot)\right\rangle, \quad \frac{\partial G}{\partial \theta}=\left\langle\left(\begin{array}{c}
i Z u(Z, \cdot) \\
i Z v(Z, \cdot) \\
i Z w(Z, \cdot) \\
0
\end{array}\right), q_{0}(\cdot)\right\rangle
$$

These inner products are evaluated using the trapezoidal rule at the collocation points of the spectral method. In the formula for $\frac{\partial G}{\partial \theta}$, we used the fact that

$$
\frac{\partial u}{\partial \theta}(z, t)=-i z u(z, t), \quad \int_{-\pi}^{\pi} \mathfrak{R}\left\{(-i Z u(Z, t))^{*} u(Z, t)\right\} d t=0,
$$

with similar formulas for $v$ and $w$. It remains to determine $\frac{\partial G}{\partial g_{0}}$ and

$\frac{\partial G}{\partial \mathfrak{R}\left(\hat{u}_{k}\right)}, \quad \frac{\partial G}{\partial \mathfrak{J}\left(\hat{u}_{k}\right)}, \quad \frac{\partial G}{\partial \mathfrak{R}\left(\hat{v}_{k}\right)}, \quad \frac{\partial G}{\partial \mathfrak{J}\left(\hat{v}_{k}\right)}, \quad \frac{\partial G}{\partial \mathfrak{R}\left(\hat{w}_{k}\right)}, \quad \frac{\partial G}{\partial \mathfrak{J}\left(\hat{w}_{k}\right)}, \quad\left(-k_{\max } \leq k \leq k_{\max }\right)$,

where $k_{\max }$ is the cutoff beyond which Fourier modes of the initial condition are set to zero. These can all be computed simultaneously by solving a single adjoint PDE as described in Appendix B.

In summary, the ACM uses adjoint methods to compute $G$ and $\nabla G$ in just two PDE solves: the nonlinear evolution equations (1d)-(1g) are solved to compute $G$ and two components of the gradient, $\frac{\partial G}{\partial Z}$ and $\frac{\partial G}{\partial \theta}$; then the non-autonomous (but linear) adjoint system (B.5) is solved to obtain the remaining components of the gradient, namely $\frac{\partial G}{\partial g_{0}}$ and those listed in (6). These are the ingredients needed to use the BFGS method to minimize $G$ and obtain a time-periodic solution. We do this repeatedly for different values of the bifurcation parameter to sweep out families of solutions. When performing numerical continuation, the approximate inverse Hessian matrix from the final iteration of the previous BFGS solve is used to initialize the inverse Hessian matrix for the next solve. As long as the continuation steps are not too large, this dramatically reduces the number of line searches required to minimize $G$ due to superlinear convergence in the BFGS algorithm when the Hessian is well approximated. 


\section{Bifurcation Sequence}

In this section, we apply the ACM to track branches of $z$-periodic solutions in the WGAML and study the sequence of bifurcations responsible for the transition from a one-pulse solution to a two-pulse solution. If $g_{0}$ is increased adiabatically, the system will transition from a stationary single-pulse solution to the period-one breather solutions, the period-two breather solutions, the chaotic solutions, and finally the two-pulse solutions.

The one- and two-pulse stationary solutions have been previously computed in the work by Kutz and Sandstede [4] and the stability of these solutions has been computed to high accuracy by Jones and Kutz [37] using the FloquetFourier-Hill method. At $g_{0}=2.404$, the single-pulse solution undergoes Hopf bifurcation. In Section 4.1, we track the branch of period-one breathers from the Hopf to the first period-doubling bifurcation. In Section 4.2, we track the period-one breathers beyond the first period doubling bifurcation to reveal an additional bifurcation that occurs at higher values of $g_{0}$. This bifurcation will not appear physically because the system is already unstable, but it reveals the existence of additional branches of solutions in the WGAML. In Section 4.3, the branch of period-two breather solutions is tracked and the loss of stability established. Finally, in Section 4.4 we discuss potential mechanisms for completing the transition to the double-pulse solution.

\subsection{Period-one breather solutions}

The first non-trivial $z$-periodic solutions are the period-one breather solutions. These solutions are created by a Hopf bifurcation of a single-pulse stationary solution at $g_{0}=2.404$. In order to apply the ACM of Section 3, an initial approximation of the limit cycle is required. From previous numerical experiments with the parameter values in (4), it was found that at $g_{0}=2.5$ a periodic solution exists and forms from noise [3]. Starting with $u(0, t)=\operatorname{sech}(t)$ and $v(0, t)=w(0, t)=0$ and evolving until $z=2000$, the system approaches that limit cycle. From this orbit, the period and phase offset were estimated and an approximation of the limit cycle with $G \sim 10^{-1}$ was obtained. The application of the ACM to this starting point reduced the error to $G \sim 10^{-25}$ and produced the solution shown in Figure 4.

Figure 4 shows the intensity of the fields in all three waveguides as a function of $z$ and $t$ for $g_{0}=2.5$. The black solid and black dashed lines show the solution at the start of the period and after half of the period has elapsed. Fifty-six intermediate values, evenly spaced in $z$, are also plotted in light-gray (green). This 

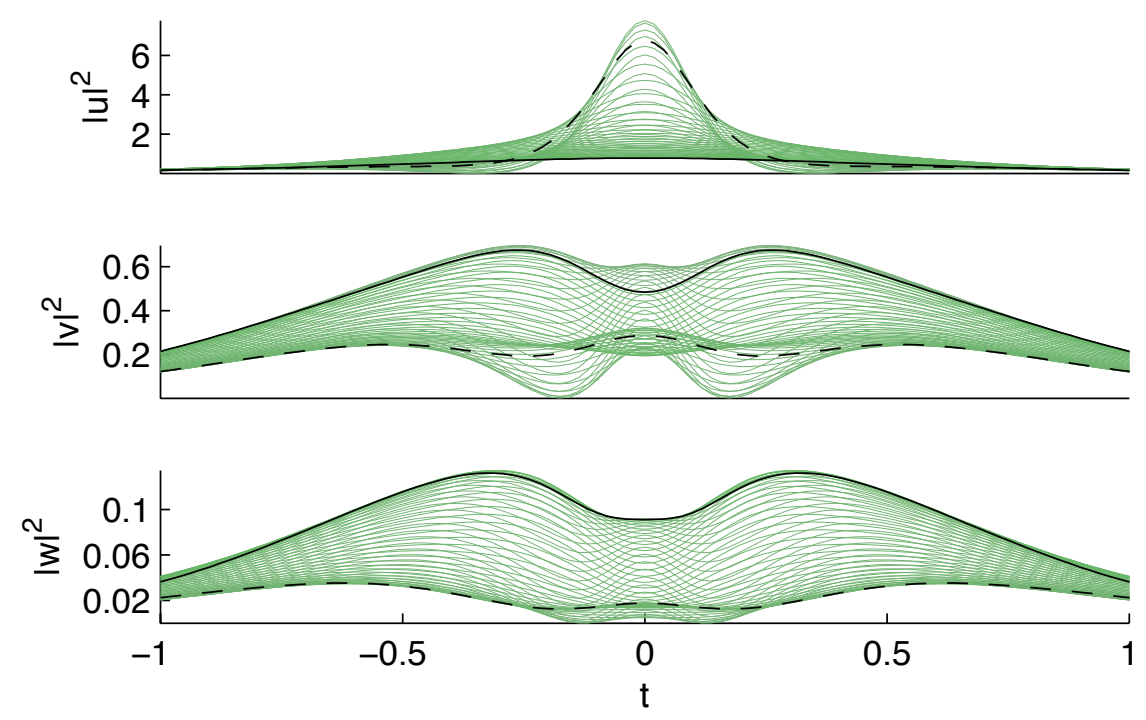

Figure 4: (Color online, two column) From top to bottom, plots of the amplitude of the 0th, 1st, and 2 nd waveguides over one period at $g_{0}=2.5$. The solid black line shows the initial condition, and the dashed black line shows the solution after a half-period has elapsed. The light-gray (green) lines show the solutions at evenly-spaced intermediate values of $z$. Note that the actual domain is from $t \in[-20,20)$ and only $t \in[-1,1]$ was plotted to highlight the region of interest.

sample solution highlights the non-trivial nature of the breather solutions. Although the 0th waveguide retains a vaguely hyperbolic-secant shape throughout the period, the 1 st and 2nd waveguides have at least two local maxima. However, despite the more complicated physical representation, the period-one breather solutions are even functions up to a translation in $t$. The source of the periodic oscillation in $z$ can be explained by the interchange of pulse energy $\left(\|u\|^{2},\|v\|^{2}\right.$, or $\|w\|^{2}$ ) between the 0th and the outer two waveguides. For the majority of the period, the 0th waveguide has a low intensity. During this time, the gain remains unsaturated and the 0th waveguide couples energy into the outer waveguides. At a certain point, this process is reversed and the outer waveguides couple energy into the 0th waveguide. This causes the 0th waveguide to attain a large peak intensity but simultaneously saturates the gain. With the gain saturated, the large amount of energy in the 0th waveguide cannot be maintained. As a result, the energy in the 0th waveguide decreases and the process repeats.

Figure 5 shows the branch of period-one breather solutions. To obtain this branch of solutions, we used a simple continuation method. From the solution at $g_{0}=2.5$, we change the period, $Z$, by a small amount and repeat the minimization process as discussed in Section 3. Linearly stable regions are shown in 

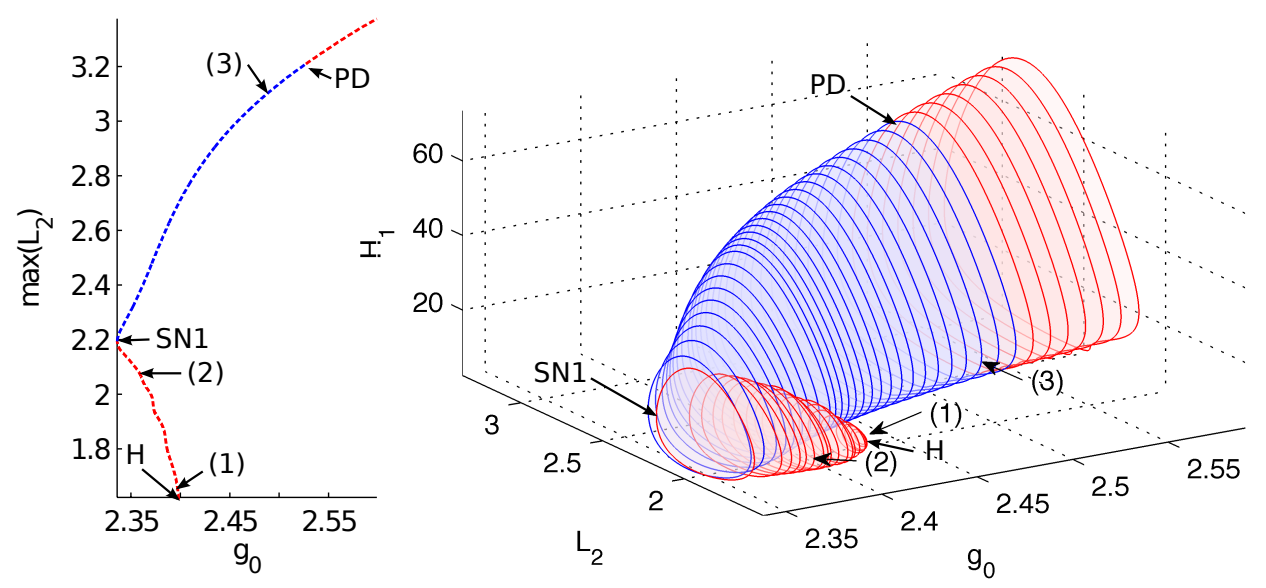

Figure 5: (Color online, two column)(Left) A two-dimensional version of the bifurcation diagram showing the maximum $L_{2}$-norm over a period vs the gain. Stable solutions are plotted in blue and unstable solutions in red. The labeled bifurcation points are shared between the 2D and 3D plots as well as Fig. 2. (Right) The bifurcation diagram of the period-one breather solutions with individual orbits are plotted in the $\left(g_{0}, L_{2}, H_{1}\right)$ coordinate system. The plotted orbits represent one out of every forty periodic orbits computed on the branch. Linearly stable orbits are shown is black (blue) and linearly unstable orbits are shown in gray (red). The solutions at points (1) and (2) are shown in Figure 7 while the solution at (3) is shown in Figure 4.

black (blue) while linearly unstable regions are shown in gray (red). In order to visualize the branch, the branch has been plotted in $\left(g_{0}, L_{2}, H_{1}\right)$ space where

$$
\begin{aligned}
& L_{2}(z)=\int_{-\infty}^{\infty}\left(|u(t, z)|^{2}+|v(t, z)|^{2}+|w(t, z)|^{2}\right) d t \\
& H_{1}(z)=\int_{-\infty}^{\infty}\left(\left|\partial_{t} u(t, z)\right|^{2}+\left|\partial_{t} v(t, z)\right|^{2}+\left|\partial_{t} w(t, z)\right|^{2}\right) d t .
\end{aligned}
$$

The bifurcation diagram is composed from roughly 2000 individual computations starting from $g_{0}=2.5$, which is labeled as (3). Each PDE computation was performed using 1024 Fourier modes to represent the solution in $t$ with a domain size of 20 and 500 steps in $z$. With this level of discretization, $G<10^{-24}$ for all points on the bifurcation diagram, but most solutions had $G \sim 10^{-25}$ or better.

There are three bifurcations that are critical for describing the period-one breather solutions: the subcritical-Hopf bifurcation that creates the branch, the saddle-node bifurcation of a limit cycle that restores stability, and the perioddoubling bifurcation that breaks symmetry and changes the stability. 


\subsubsection{Subcritical Hopf bifurcation}

The subcritical-Hopf bifurcation, shown in Figure 5 at (1), is where the single-pulse stationary solutions bifurcates and creates the branch of period-one breather solutions. Because this is a subcritical bifurcation, for $g_{0}<2.404$ we have both an unstable period-one breather solution and a stable single-pulse solution. Although the existence of the Hopf bifurcation has been initially shown by both Kutz and Standstede [3] and shown with high numerical accuracy by Jones and Kutz [37], it was not known whether the Hopf bifurcation was super- or sub-critical. By tracking the branch of periodic solutions back to the bifurcation point, we have demonstrated the bifurcation is a subcritical Hopf bifurcation.

\subsubsection{Saddle-node bifurcation}

The next bifurcation that occurs is the saddle-node bifurcation of a limit cycle near $g_{0}=2.325$. This bifurcation restores the stability of the period-one branch of solutions as can be seen in Figure 5. The segment of the branch between the saddle-node bifurcation and the period-doubling bifurcation around $g_{0}=2.523$ is the only region where the period-one breather solutions are stable. This segment of the branch overlaps with the stable regime of the single-pulse solutions. Indeed, for $g_{0} \in(2.325,2.404)$ there are at least three potential solutions, the stable stationary single-pulse solution, the stable high-amplitude breather solution (shown in blue), and the unstable low-amplitude breather solution (shown in red). In simulations starting from noise, the stationary solution is most likely to appear. This can be argued from an energy perspective. This energy argument is based on physical principals as well as a number of numerical and experimental results where it has been observed that the system generically evolves to the lowest energy solution. However, for infinite dimensional systems, there is no rigorous proof for this behavior, and to prove so is beyond the scope of the present work.

Of the three solutions available, the low amplitude breather solution has the lowest $L_{2}$ norm at certain values of $g_{0}$ but is unstable. Of the two stable solutions, the stationary single-pulse solution has a lower $L_{2}$ norm than the periodone breather solution for all $z$. The single-pulse solution is more energetically favorable than the breather solution and therefore more likely to occur. With a similar argument, the stationary two-pulse solutions are also unlikely to form from noise. However because the single-pulse solutions, period-one breathers, and double-pulse solutions are all stable, there is a non-zero probability of any of those solutions appearing when starting from white-noise initial conditions. 

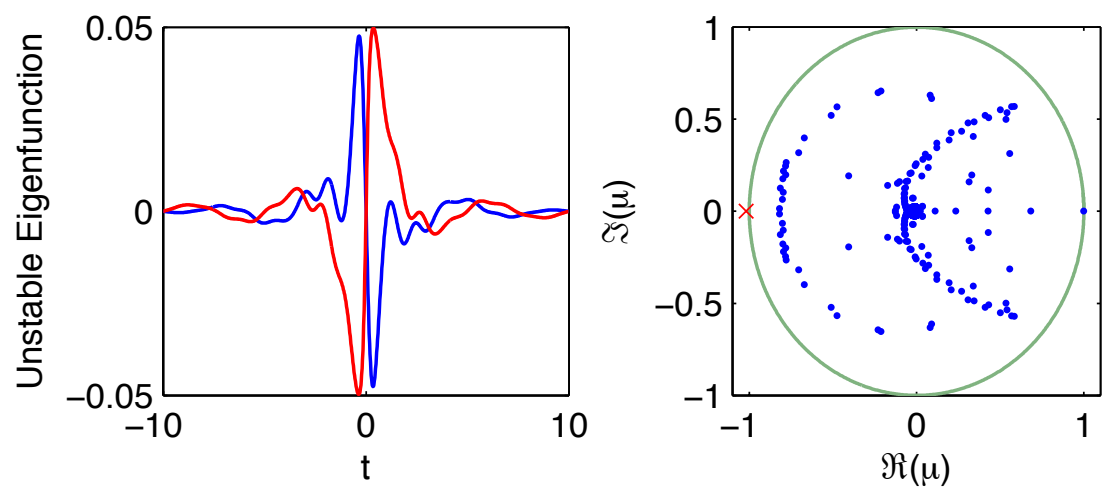

Figure 6: (Color online, two column) On the left, the real and imaginary parts of the bifurcation eigenfunctions of the 0th waveguide are shown in black (blue) and gray (red) respectively. These modes are associated with a period doubling bifurcation indicated by a Floquet multiplier crossing the unit circle at $\mu_{\mathrm{pd}}=-1$ in the right figure. Multipliers in or on the unit circle are indicated with a black (blue) dot. Multipliers outside of the unit circle are indicated with a gray (red) $x$.

\subsubsection{Period-doubling bifurcation}

The final bifurcation shown in Figure 5 is the period-doubling bifurcation that occurs at $g_{0}=2.523$. Figure 6 shows the unstable eigenfunction at $z=0$ and the associated Floquet multipliers. The single unstable multiplier at $\mu_{\mathrm{pd}}=-1$ demonstrates that this is a period-doubling bifurcation [38]. Furthermore, the unstable eigenfunction associated with this bifurcation is odd. From (B.3), it can be shown that for any odd $\dot{u}(t, 0), \dot{v}(t, 0)$, and $\dot{w}(t, 0)$ with $\dot{g}_{0}=0$, which is exactly what we have in computing the Floquet multipliers, $\dot{u}(t, z), \dot{v}(t, z)$ and $\dot{w}(t, z)$ remain odd for all $z$. Therefore, the unstable eigenfunction breaks symmetry at all points on the orbit. We believe that the symmetry breaking due to this bifurcation is responsible for the translating behavior that appears when the period-one breather goes unstable.

\subsubsection{Computing the stability of periodic solutions}

In order to compute the Floquet multipliers of this system, the monodromy matrix [38] was computed for the linearized system in (B.3) with $\dot{g}_{0}=0$ and the ODE for $\dot{g}_{0}$ dropped. This term is zeroed out because for any given periodic solution $g_{0}$ is fixed. The complex solutions of (B.3) were rewritten as a real system of twice the size by splitting the solutions into real and imaginary parts. If the matrix $X(z)$ is the fundamental solution matrix of this system at $z$, then the monodromy matrix is

$$
M=X\left(z_{0}+Z\right) X^{-1}\left(z_{0}\right)
$$


Because the linearized operator in (B.3) is non-autonomous, $X\left(z_{0}+Z\right)$ must be computed explicitly by solving (B.3) for the set of initial conditions implicitly described by $X\left(z_{0}\right)$. For the multipliers computed above, $X\left(z_{0}\right)$ was represented in the Fourier basis and $\hat{X}\left(z_{0}\right)=$ id where the hat represents the discrete Fourier transform acting on the columns of $X\left(z_{0}\right)$. In order to compute $u(z, t)$ at intermediate timesteps, the same Hermite interpolation and semi-implicit time-stepper used to solve the adjoint equation were also used to compute the monodromy matrix. Due to the large number of degrees of freedom in the system, this is an expensive computation. However, the majority of the time is spent computing $X\left(z_{0}+Z\right)$, and this portion of the computation is easily parallelizable. With the monodromy matrix assembled, MATLAB was used to compute the eigenvalues.

The linearized equations in (B.3) has three multipliers where one can show $\mu_{1}=1$, one from the phase-condition, one from phase-invariance, and one from translational invariance in $t$. This approach recovers these multipliers with $\mid \mu_{1}-$ $1 \mid<10^{-4}$. Although this approach is too computationally intensive to be used on all solutions, it was used to compute the stability of solutions where a bifurcation was suspected to occur.

\subsubsection{Deformation of the period-one breathers}

In addition to the bifurcations, the deformations of the period-one breather solutions as we travel along the branch of solutions result in breathers that look qualitatively different at different values of $g_{0}$. As we travel away from the Hopf bifurcation along the branch of solutions, the $L_{2}$ norm and the $H_{1}$ norms of solutions increase monotonically, and the solutions deform steadily from the hyperbolic secant solutions of (3) to the nontrivial breather solutions in Figure 4 indicated by (3) in Figure 5.

Figure 7 plots the solutions at (1) and (2) in Figure 5 with $g_{0}=2.398$ and 2.37, respectively, and highlights the deformation of the breathers. The solution at $g_{0}=2.398$ is a small perturbation away from the single-pulse solution and can be treated as the single pulse solution plus a small $z$-periodic perturbation; thus, all three waveguides have intensities that resemble a hyperbolic secant. Even at this early stage, it is apparent that when $\|u\|$ is maximal, both $\|v\|$ and $\|w\|$ take on their minimal values. Therefore, the dynamic interchange of energy between waveguides is already occurring, if only on a relatively small scale.

Further along the branch at (2) in Figure 5, the magnitude of the oscillation has grown in all three of the waveguides. In addition, the breathing that occurs in the 2nd waveguide now includes the characteristic local minima at $T=0$ that was observed in both waveguides 1 and 2 at $g_{0}=2.5$. These low-amplitude breather solutions provide the intermediate steps between the Hopf bifurcation 
Breather Solution at 1
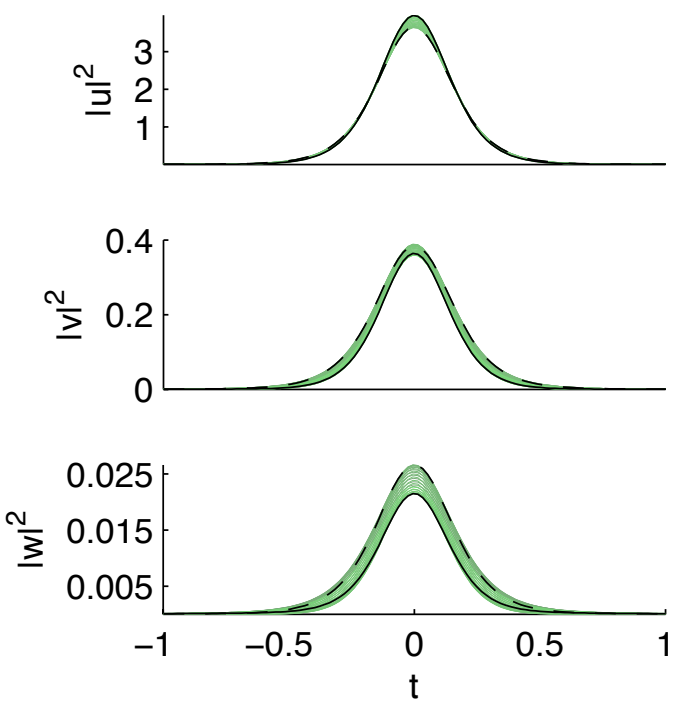

Breather Solution at 2
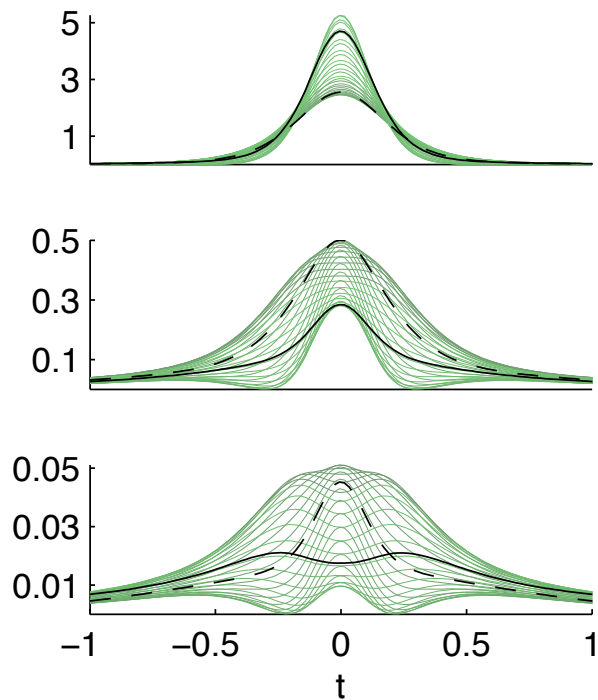

Figure 7: (Color online, two column) Plot of the breather solutions at the points labeled (1) and (2) in Figure 5 on the left and right respectively. The solid black line shows the initial condition and the dashed black line shows the solution after a half-period has elapsed. The gray (green) lines show the evolution of the solution in evenly spaced intervals. The left set of plots was taken directly after the Hopf bifurcation, and the right set of plots was taken from the unstable region after the first fold of the limit cycle. Note that the actual domain is from $t \in[-20,20)$ and only $t \in[-1,1]$ was plotted to highlight the region of interest.

and the breather solutions observed in previous works [3]. In both cases, these solutions have not been previously observed because they are linearly unstable. However, the ACM allows the tracing of these unstable solutions so it is not a barrier in this case.

Overall, the period-one breather solutions are critical because they remove the stability of the stationary solutions and bifurcate with an odd mode. The odd mode breaks the even symmetry previously inherent in both the stationary and breather solutions. This symmetry breaking explains how the translations observed in [4] could occur even when the initial conditions are even functions. Even if the initial condition is truly even, experimental noise or numerical roundoff error will excite the odd mode, which then grows and becomes nonnegligible. Furthermore, the structure of the period-one breather branch at low amplitudes, which is initiated by a subcritical Hopf bifurcation and later gains stability through a saddle-node bifurcation, explains the sudden jump in energy that occurs when $g_{0}$ is adiabatically increased through $g_{0}=2.404$. Therefore al- 

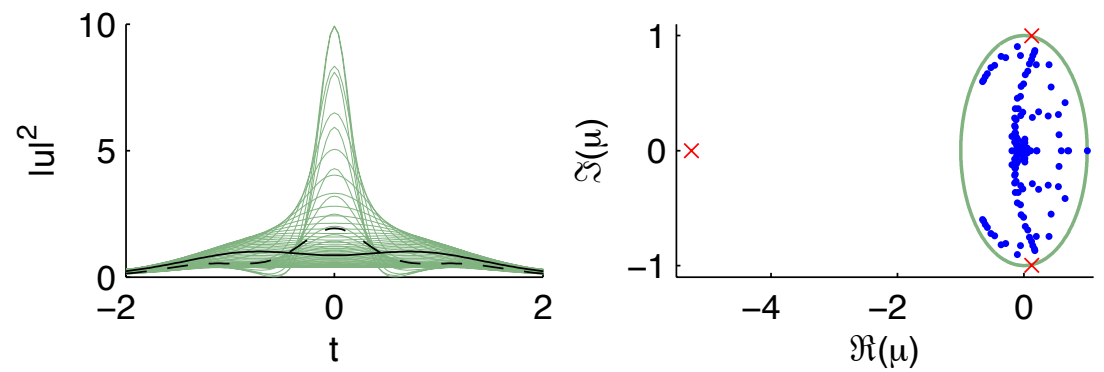

Figure 8: (Color online, two column) On the left is the plot of the amplitude of the breather solution at $g_{0}=2.85$ in waveguide 0 , and on the right are the Floquet multipliers. Floquet multipliers outside the unit circle are denoted with a grey (red) $x$, and multipliers inside or on the unit circle are denoted with a black (blue) dot. There are multipliers outside the unit circle: $\mu_{\mathrm{pd}}=-5.825$ and $\mu_{\mathrm{ns}}=0.1217 \pm 0.9984 i . \mu_{\mathrm{pd}}$ is associated with an odd eigenfunction and has remained outside the unit circle since the period-doubling bifurcation. The additional pair, $\mu_{\mathrm{ns}}$ corresponds to a torus (Neimark-Sacker) bifurcation that occurs near $g_{0}=2.8$.

though the unstable limit cycles revealed by the adjoint method will never appear in direct numerical simulation from noise, they are necessary to fully explain the dynamics that appear in such simulations.

\subsection{Torus bifurcation of period-one breathers}

The branch of period-one breather solutions extends beyond the period-doubling bifurcation for values of $g_{0}>3.0$ and does not appear to terminate. In the full PDE, these solutions are not stable and therefore will not appear in simulations starting from noise. However, as stated in the previous section, the perioddoubling bifurcation is caused by an odd bifurcating function and does not appear in systems constrained to be even, such as the one studied in [6]. The solutions that appear at larger values of $g_{0}$ are qualitatively similar to those in Section 4.1; the 0 th waveguide resembles an oscillating hyperbolic secant while the 1 st and 2nd waveguides have multiple local maxima. In an even-constrained system, the branch of solutions remains stable until $g_{0} \approx 2.8$ where a torus (Neimark-Sacker) bifurcation occurs [38].

Figure 8 shows the solution at $g_{0}=2.8$ and the Floquet multipliers of the solution. The solution itself is still qualitatively similar to period-one breathers at lower values of $g_{0}$. The difference is that an additional pair of Floquet multipliers has crossed out of the unit circle. As shown on the right in Figure 8, there are three unstable multipliers. The eigenfunction associated with the multiplier at $\mu_{\mathrm{pd}}=-5.825$ has existed since the period-doubling bifurcation occurred. The 
new complex-conjugate pair of multipliers, $\mu_{\mathrm{ns}}=0.1217 \pm 0.9984 i$, signifies the presence of a torus bifurcation. The eigenfunctions associated with these multipliers are even at $z=0$ and from (B.3) can be shown to be even for all $z$. Therefore in the even-constrained system, it is the torus bifurcation that is responsible for the loss of stability of the period-one breathers.

In an unconstrained version of the WGAML, this bifurcation does not appear in any meaningful way. Any perturbation will grow primarily in the direction of the eigenfunction with $\mu=-5.825$, and the presence of this additional pair can be neglected. It is only in even-constrained systems where the torus bifurcation is responsible for the loss of stability of the period-one breathers. A reduced order model of the even-constrained system was studied in [6]. In that lowdimensional model, the torus bifurcation was responsible for eventual route to chaos in the system. Here we have shown that the same torus bifurcation occurs in this system. Although the adjoint method cannot track the resulting quasiperiodic solutions, this indicates that the low dimensional model in [6] may be correct.

\subsection{Period-two solutions}

The period-two branch of solutions bifurcates from the period-one solutions at $g_{0}=2.523$. Because the bifurcating eigenfunctions are odd, unlike the periodone breathers the period-two breathers are neither even nor odd. Figure 9 shows a sample solution taken at $g_{0}=2.527$. The clearest difference between the periodone breather and the period-two breather is that the zeroth waveguide reaches its maximum intensity in two places, near $t=-0.5$ and $t=0.5$. A similar oscillation occurs in waveguides 1 and 2. This shift in $t$ is the reason for the doubling of the period. In Figure 9, the solid and dashed lines correspond to $z=0$ and $z=Z / 2$, respectively. These two lines help show that the solution at $z=0$ and the solution at $z=Z / 2$ are reflections of each other across $t=0$. Due to the reflectional symmetry in the problem, the solution spends exactly half the period shifted towards negative values of $t$ and the other half shifted towards positive values of $t$.

From this initial point, we can track the branch of solutions using the period, $Z$, as the continuation parameter again. The bifurcation diagram in Figure 10 shows the period-two solution in $\left(g_{0}, L_{2}, H_{1}\right)$ space where the $L_{2}$ and $H_{1}$ norms are defined in (7). The stable orbits are shown in black (blue) while the unstable orbits are shown in gray (red). The branch of period-two solutions begins near the point labeled (a) in Figure 10. Immediately after this bifurcation, the periodtwo solutions are stable until $g_{0}=2.527$. Notice that the region for which the 

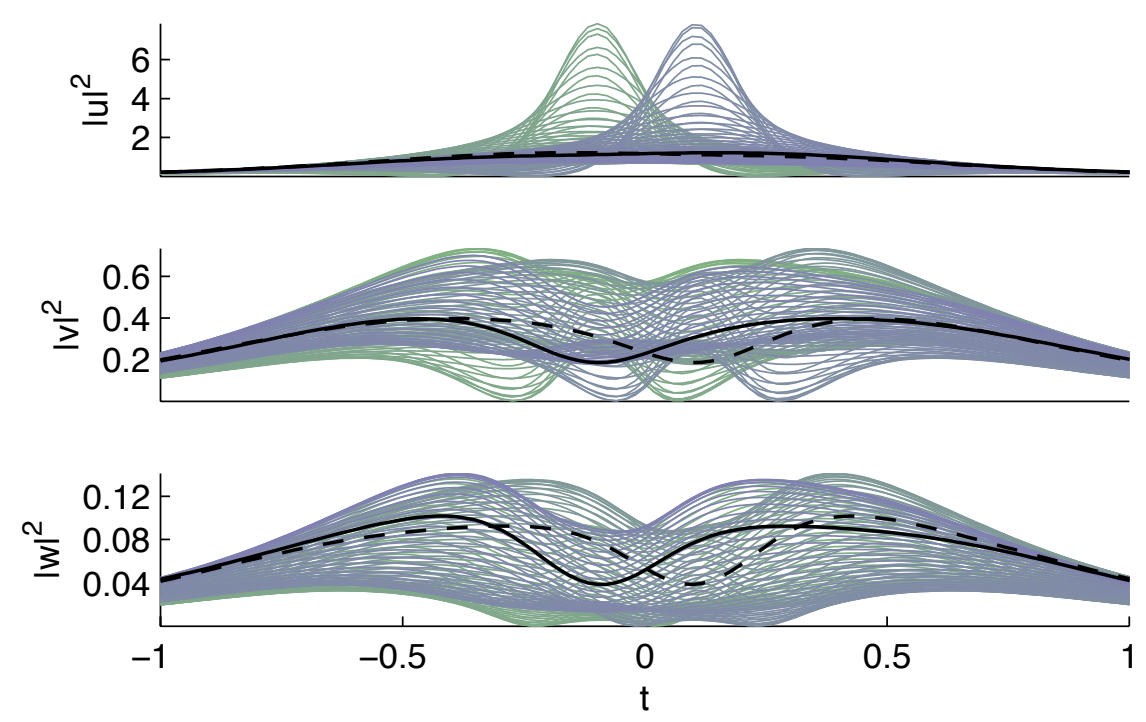

Figure 9: (Color online, two column) Example of a stable, period-doubled solution. The symmetry breaking of the period-doubling bifurcation has generated a double-peak structure in all three waveguides. The solid black line shows the initial condition and the dashed black line shows the solution after a half period has elapsed. The solution at evenly spaced intermediate times are denoted with gray lines. ( The first half-period is shown in blue and the second half-period is shown in green). Note that the actual domain is from $t \in[-20,20)$ and only $t \in[-1,1]$ was plotted to highlight the region of interest.

period-two solutions are stable is very small with $\Delta g_{0} \approx 0.002$. For this reason, the period-two solutions rarely appear when starting from noise.

The stable branch of period-two breathers is terminated by a saddle-node bifurcation around $g_{0}=2.527$. At this point a Floquet multiplier exits the unit circle through $\mu=1$, and there is a fold in the branch of solutions. In a certain sense, this fold is similar to the subcritical Hopf bifurcation of the periodone branch. The branch of period-two solutions extends below the value of $g_{0}$ where the branch began, and the solutions are unstable during this time. The key difference between the period-two branch and the period-one branch is that an additional bifurcation occurs on this low amplitude branch. At this point, an additional multiplier exits the unit circle through $\mu=1$, resulting in two multipliers outside of the unit circle. Although the second saddle-node bifurcation occurs at $g_{0}=2.251$, which brings one multiplier inside the unit circle, the resulting large-amplitude orbits are still unstable. This extra bifurcation is the cause of the vastly different regions of stability despite the qualitative resemblance of solution branches. 

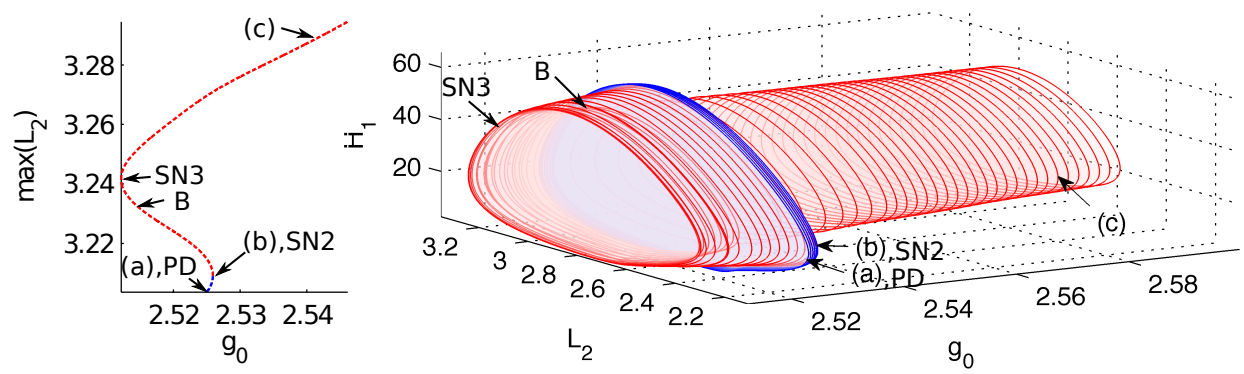

Figure 10: (Color online, two column) (Left) A two-dimensional version of the bifurcation diagram showing the maximum $L_{2}$-norm over a period vs the gain. Stable solutions are plotted in blue and unstable solutions in red. The labeled bifurcation points are shared between the $2 \mathrm{D}$ and 3D plots as well as Fig. 2. (Right) The bifurcation diagram of the period-two solutions to the WGAML. Individual orbits are plotted in $\left(g_{0}, L_{2}, H_{1}\right)$ space and each plotted orbit represents one of every thirty solutions computed on the branch. Linearly stable solutions are plotted with black (blue) edges while unstable orbits are plotted with gray (red) edges.

It appears there are no stable branches of either period-one or period-two breathers beyond this point in the bifurcation diagram. The branch of smallamplitude period-two solutions could potentially be a source of additional branches of periodic solutions. However, the resulting branch of solutions would still be unstable since there remains an additional unstable eigenfunction. Following this branch using the adjoint numerical continuation proved to be unsuccessful, thus the nature of the bifurcation and its co-dimension remains an open question meriting further investigation. To our knowledge, there have been no observations of other periodic solutions that could be associated with a new unknown branch of solutions.

Figure 11 in parts (a), (b), and (c) shows sample solutions at varying points along the branch of solutions. Directly after the period-doubling bifurcation, at (a), the period-two solution is only a slight perturbation away from the periodone solution, and the peaks are indistinguishable to the eye. Further along the branch at (b), the separation between the peaks increases. This solution is taken just below $g_{0}=2.527$ and represents the largest separation that could occur while still keeping the solution stable. At point (c), the pulses are now separated by $\Delta t=1$. This trend continues as $g_{0}$ increases beyond point (c). In addition to the increased separation of the points of maximum intensity, the peak intensity decreases as we get further from the period-doubling bifurcation. Despite that, the maximum value of the $L_{2}$ norm increases monotonically the further one gets from the period-doubling bifurcation. On the other hand, the maximum value of the $H_{1}$ norm decreases monotonically at the same time. Therefore, referring back 


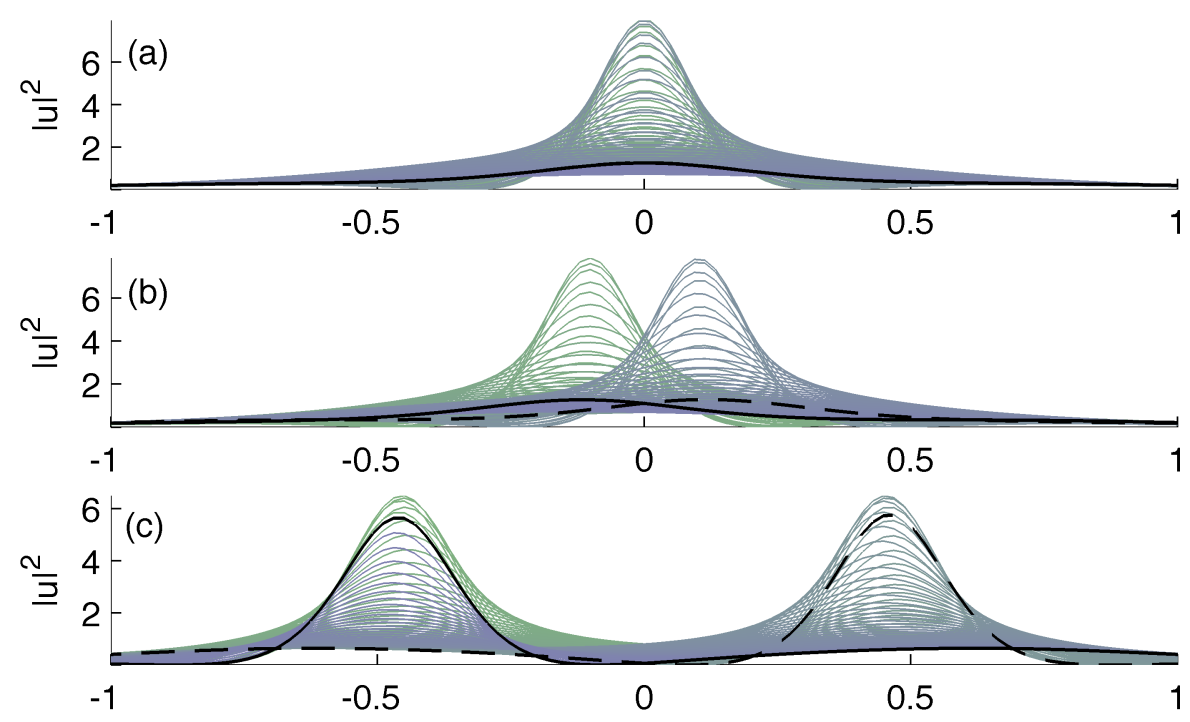

Figure 11: (Color online, two column) Plots of solutions at the three selected points indicated by (a), (b), and (c) in Figure 10. The solid-black and dashed-black lines show the intensity in the 0 th waveguide at $z=0$ and $z=Z / 2$. Intermediate times are plotted in gray (the first half-period is shown in blue and the second half-period is shown in green). Note that the actual domain is from $t \in[-20,20)$ and only $t \in[-1,1]$ was plotted to highlight the region of interest.

to Figure 10, the period-two solutions are encircled by the period-one solutions in the $L_{2}-H_{1}$ plane. The period-two solutions in turn encircle the two-pulse solutions.

The period-two solutions themselves play a relatively minor role in the transition from the single-pulse to double-pulse solutions. The region of $g_{0}$ where the solutions both exist and are stable, $g_{0} \in(2.523,2.527)$, is small compared to the regions where the stationary solutions or even the period-one breather solutions are stable. Nonetheless, tracking the branch of period-two breathers suggests that there are no remaining period-one or period-two breathers in the WGAML beyond $g_{0}=2.527$.

\subsection{Global Bifurcation Structure}

As described in previous sections, the WGAML has a variety of solutions and bifurcations when $g_{0}$ is increased. In particular, starting on the one-pulse branch, the solutions undergo the following sequence of bifurcations: the onepulse stationary solution loses stability and bifurcates to an unstable breather, which undergoes a fold bifurcation and becomes stable. This stable periodic so- 
lution loses stability and bifurcates to a period-two breather, which eventually becomes unstable. After this last bifurcation, additional periodic or stationary solutions were not found. In Figure 12, we summarize our findings in a combined bifurcation diagram in which the stationary and periodic solutions are projected onto the three dimensional $\left(g_{0}, L_{2}, H_{1}\right)$ space. After this projection, the stationary solutions become one-dimensional curves and periodic solutions become two-dimensional closed manifolds. The gray plane indicates the value of $g_{0}^{u}=2.527$ at which the period-two solution becomes unstable. Our study suggests that from this point on, there are no local bifurcations that result in stable stationary or periodic solutions. We conjecture that there are no stable stationary or periodic solutions near the one-pulse solutions (other than the one-pulse solutions themselves). However, outside of a neighborhood of this set, breathers and multi-pulse solutions (such as the two-pulse solution) exist and can be stable.

Indeed the two-pulse solution exists and is stable for values of $g_{0}>g_{0}^{u}$ and one would expect all trajectories to be attracted to it. However, numerical simulations and experiments indicate a more subtle behavior. Solutions initiated in the neighborhood of the two-pulse solution are attracted to it, while solutions initiated in the neighborhood of the one-pulse solution, or in the vicinity of a zero solution (low-amplitude white noise), exhibit a typical behavior of intermittent spatio-temporal chaos expressed as translations in $t$ and non-periodic behavior in $z$. For some trajectories, such a behavior is transient, and eventually the trajectory is attracted to the two-pulse solution. As $g_{0}$ is increased, we observe that more initial conditions are attracted to the double-pulse and the transients become shorter. For $g_{0}=g_{0}^{u}+\varepsilon$ such transients can be very long and numerical simulations cannot determine whether the attractor for generic white noise initial data is the two-pulse, or if there exists a trapping region in which a chaotic attractor reigns. In the latter case the trajectory will hover indefinitely between one-pulse, period-one and period-two unstable solutions.

In Figure 13 and Figure 14, we project a few typical trajectories, stationary solutions, and relevant periodic orbits in terms of $\left(g_{0}, L_{2}, H_{1}\right)$ for $g_{0}=2.3,2.5$, and 2.6. The trajectories are denoted in light gray and have low-amplitude white noise initial condition. At $g_{0}=2.3$, the solution is rapidly attracted to the stable one-pulse solution denoted by the black (blue) dot. At $g_{0}=2.5$, the one-pulse solution is unstable and the trajectory is attracted to the period-one breather solution, which is shown in black (blue). Lastly for $g_{0}=2.6>g_{0}^{u}$, the trajectory eventually is attracted to the stationary two-pulse solution. Prior to that, the solution jumps chaotically between the period-one breather solution shown in black (green) and the period-two breather solutions shown in gray (blue). 


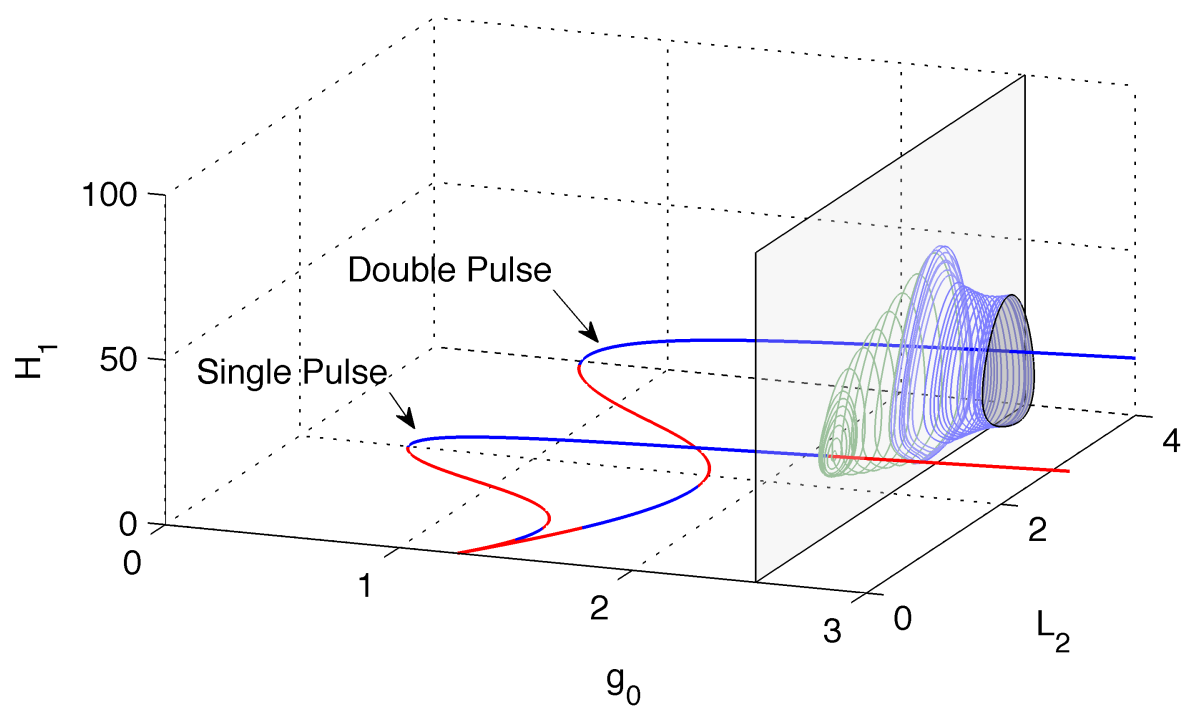

Figure 12: (Color online, two column) Bifurcation diagram of the stationary and breather solutions of the WGAML. The stationary solutions are indicated by thick lines. Solid (blue) lines indicate spectrally stable solutions while dotted (red) lines represent solutions that are spectrally unstable. The period-one limit cycles are shown in light-gray (green) and the period-two in darkgray (light-blue). The gray plane at $g_{0}=2.527$ represents the largest value of $g_{0}$ for which any of the limit cycles are stable. The gray shaded region indicates the interior of the limit cycle, and it should be noted that the stable two-pulse solution remains within the interior of the limit cycle near the transition value of $g_{0}$.

By continuing the branch of period-two solutions beyond $g_{0}^{u}$ and projecting individual solutions onto the three dimensional bifurcation diagram can shed light on this behavior. We detect in the bifurcation diagrams in Figures 10 and 12 a change in the shape of the period-two solution at $g_{0}=2.527$. Specifically, it is contracted toward the double pulse solution. Indeed the plot of its evolution over the full period (bottom plot in Figure 11) suggests that it becomes more like the double pulse solution by having two separated pulses. Combining our findings, we conjecture that there are two possibilities for the source of chaotic behavior in the multi-pulse transition. One possibility is that there is a trapping region that includes the neighborhood of the one-pulse solution and extends to the neighborhood of the zero solution. A global bifurcation eventually opens that region and the change in shape of the period-two solution might reveal this bifurcation. Another possibility is that as $g_{0}$ is increased, the two-pulse solution and its basin of attraction intersect the neighborhood of the one-pulse solution. 


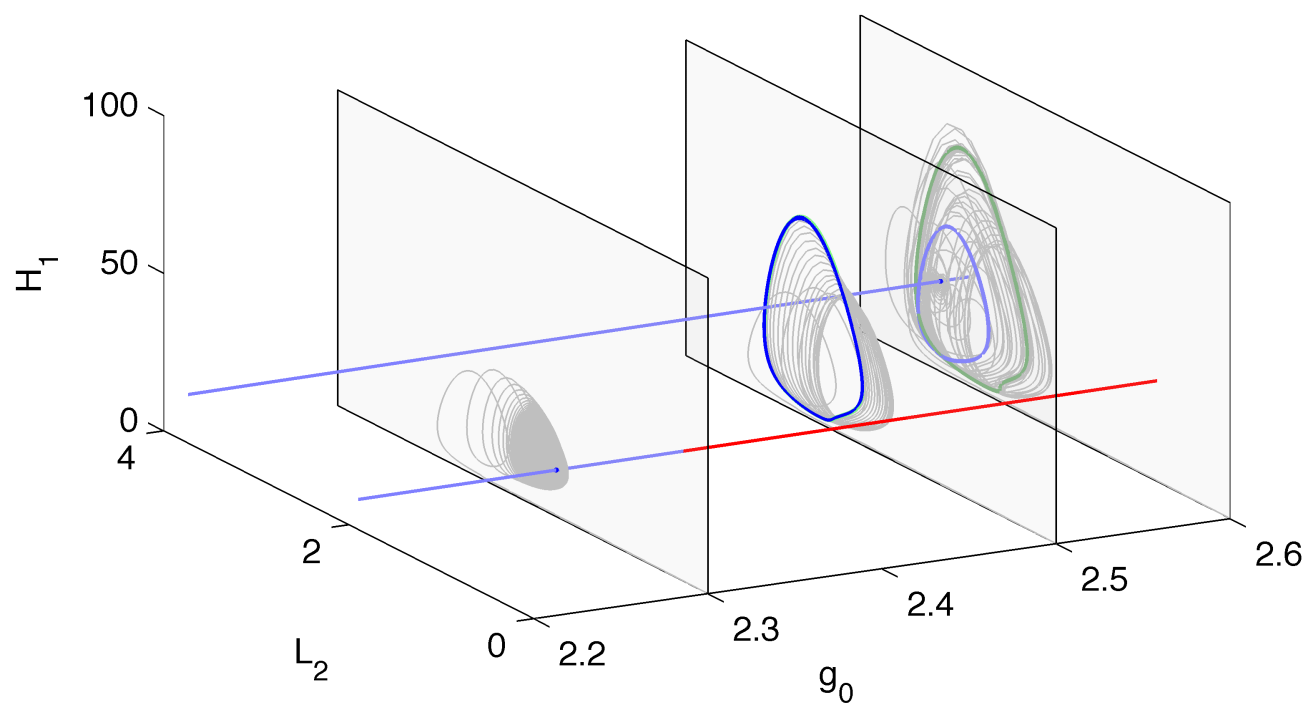

Figure 13: (Color online, two column) Plot of the trajectories of the solutions (projected onto $L_{2}$ and $H_{1}$ ) for $g_{0}=2.3,2.5$ and 2.6 starting with low-amplitude initial conditions. The long time behavior of the trajectories are shown in black (blue). The light gray lines show the complete evolution of the solutions from the initial conditions. The gray (light-blue) lines show the stable single- and double-pulse solutions, while the light-gray (red) lines show the unstable singlepulse solutions. The dark gray lines show other limit cycles at the same value of $g_{0}$, such as the period-one breathers (green) and period-two breathers (light-blue).

As this intersection occurs, the trajectories will asymptotically be attracted to the two pulse solution. We have observed similar multi-pulsing phenomena in the transition from a general $N$-pulse solution to an $N+1$-pulse solution. This suggests that our study in this paper will be relevant to the study of the multipulsing transition sequence in mode-locked laser systems. Given that the only transitions observed experimentally $[1,7,20,21]$ and theoretically $[8,9,20,21]$ are from $N$ to $N+1$ pulses, the method developed characterizes this fundamental behavior.

\section{Conclusion and Experimental Verification}

A highly accurate $\left(G<10^{-24}\right)$ characterization of the bifurcation structure of the multi-pulsing instability in a laser cavity is performed by employing the ACM on the WGAML model. The analysis shows that a subcritical-Hopf bifurcation on the branch of single-pulse solutions creates the branch of period-one breather 

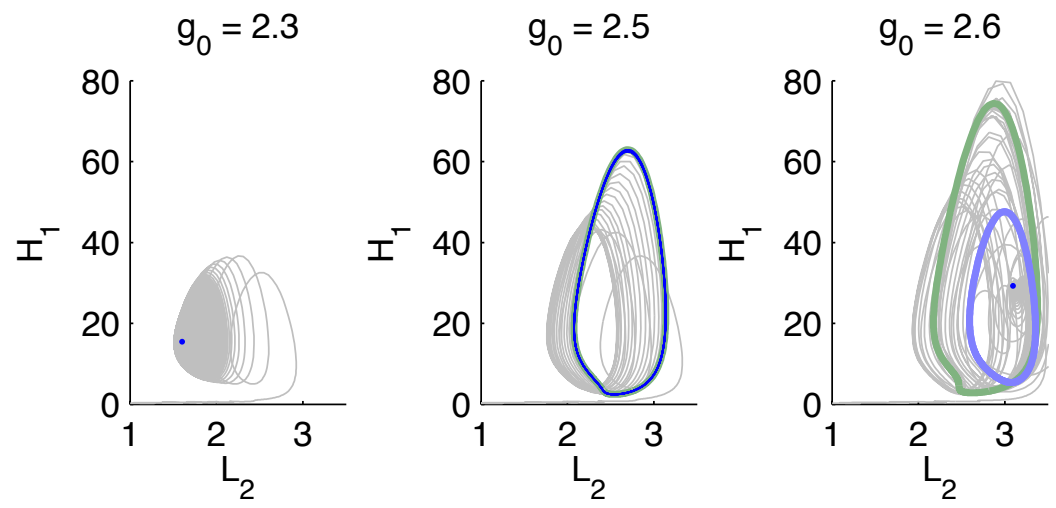

Figure 14: (Color online, two column) Plot of the trajectory of the solution starting from lowamplitude white noise for $g_{0}=2.3,2.5$, and 2.6, respectively. The solution trajectory is shown in light gray. The dark gray (green and light-blue) lines show the period-one and period-two limit cycles for values of $g_{0}$ where they exist. The black (blue) points or curves denote the long time behavior of the trajectory. At $g_{0}=2.3$, the stationary single-pulse solution is the attractor. At $g_{0}=2.5$, the period-one breather solution is the limiting solution. At $g_{0}=2.6$, the chaotic translating double-pulse solution gives the long time behavior.

solutions. A period-doubling bifurcation on that branch creates the branch of period-two breather solutions. A pair of saddle-node bifurcations and a third bifurcation which the authors have not yet classified are responsible for the branch of period-two breathers becoming unstable for $g_{0}>2.527$. Therefore, when cavity gain is large, the period-two breather solutions are destabilized, which leads to the chaotic-like behavior observed in this system.

For even initial data, the subcritical-Hopf bifurcation is instead followed by a torus (Neimark-Sacker) bifurcation, leading to quasi-periodicity in the laser cavity. This affirms the results of previous work using low-dimensional POD analysis [6]. Further increase of the cavity gain results in stable, two-pulse operation in both cases. This transition behavior is generic for $N$ to $N+1$ pulses in the laser cavity, thus confirming mode-locking experiments $[1,7,20,21]$ and theory $[8,9,20,21]$. The demonstrated transition repeats itself for the 2- to 3-pulse transition, the 3- to 4-pulse transition and so on as demonstrated in [4].

These numerical predictions, although specific to the WGAML model, corroborate experimental and theoretical evidence for such a series of bifurcations. Clearly the most important connection to make is with direct experimental observations of mode-locked laser cavities. Although early observations demonstrated the multi-pulsing transition (see, for instance, Namiki et al. [7]), more 
careful experiments near the multi-pulsing transition point were not performed for another decade [20, 21]. The recent experimental observations in 2009 by the Wise group at Cornell University [20] and in 2004 by the Grelu group at the University of Bourgogne [21] both carefully considered the multi-pulsing transition points and found all the key features of the bifurcation diagram constructed here. These results were achieved despite the fact that the Cornell group used a carbon nanotube for saturable absorption while the Bourgogne group used nonlinear polarization rotation. Thus although neither experiment used a waveguide array for the mode-locking process, the transition sequence from a steady-state to a Hopf state to period doubled states to quasi-periodicity to multi-pulsing was clearly observed in both experiments. This strongly suggests that the multi-pulsing bifurcation sequence considered here is universal in nature for laser cavities.

In addition to experimental work, several theoretical models have been proposed to explain the multi-pulsing transition. One of the earliest models was by Namiki et al. [7] in which a clear prediction was made about the stability and existence of multi-pulse states. However that analytical formulation did not address the transition behavior. A recent extension of that work, where the gain and loss dynamics involved a discrete mapping [8], has also successfully predicted the transition phenomenon observed in the recent experiments highlighted in the previous section, i.e. steady-state - Hopf - period doubling - torus - multipulsing. Furthermore, a number of computational studies have been performed on mode-locked lasers where evidence of the above bifurcation sequence was observed via direct numerical simulation $[4,20,21]$.

The results of this manuscript agree with and extend the existing experimental and theoretical efforts. In particular, this is first time that the bifurcation structure, including the explicit determination of the unstable solutions and of the bifurcations that occur, has been computed in the PDE. Although this analysis is specific to the waveguide array mode-locking model, due to the ubiquity of the multi-pulsing transition it is conjectured that a similar sequence of bifurcations is responsible for the multi-pulsing transition in a large number of experimentally realizable mode-locked laser cavities.

\section{Acknowledgements}

J. N. Kutz acknowledges support from the National Science Foundation (NSF) (DMS-1007621) and the US Air Force Office of Scientific Research (AFOSR) (FA9550-09-0174). J. Wilkening was supported in part by the Director, Office of Science, Computational and Technology Research, U.S. Department of Energy under Contract No. DE-AC02-05CH11231, and by the National Science 


\section{Foundation through grant DMS-0955078.}

\section{References}

[1] H. Haus, Mode-locking of lasers, IEEE J. Sel. Top. Quant. Elec 6 (2000) 1173-1185.

[2] J. N. Kutz, Mode-locking of fiber lasers via nonlinear mode-coupling, in: N. N. Akhmediev, A. Ankiewicz (Eds.), Dissipative Solitons, Springer-Verlag, Berlin, 2005.

[3] J. Proctor, J. N. Kutz, Theory and simulation of passive mode-locking with waveguide arrays, Opt. Lett. 13 (2005) 2013-2015.

[4] J. N. Kutz, B. Sandstede, Theory of passive harmonic mode-locking using waveguide arrays, Opt. Exp. 16 (2008) 636-650.

[5] J. Proctor, J. N. Kutz, Averaged models for passive mode-locking using nonlinear modecoupling, Math. Comp. in Sim. 74 (2007) 2471-2481.

[6] M. O. Williams, E. Shlizerman, J. N. Kutz, The multi-pulsing transition in mode-locked lasers: A low-dimensional approach using waveguide arrays, J. Opt. Soc. Am. B 27 (2010) 2471-2481.

[7] S. Namiki, E. P. Ippen, H. A. Haus, C. X. Yu, Energy rate equations for mode-locked lasers, J. Opt. Soc. Am. B 14 (1997) 2099-2111.

[8] L. Feng, P. K. A. Wai, J. N. Kutz, A geometrical description of the onset of multi-pulsing in mode-locked laser cavities, J. Opt. Soc. Am. B 27 (2010) 2068-2077.

[9] J. N. Kutz, Mode-locked soliton lasers, SIAM Rev. 48 (2006) 629-678.

[10] F. Friberg, A. Weiner, Y. Silberberg, B. Sfez, B. Smith, Femtosecond switching in a dualcore-fiber nonlinear coupler, Opt Lett 13 (1988) 904-906.

[11] H. Eisenberg, Y. Silberberg, R. Morandotti, A. R. Boyd, J. S. Aitchison, Discrete spatial optical solitons in waveguide arrays, Phys. Rev. Lett. 81 (1998) 3383-3386.

[12] A. Aceves, C. D. Angelis, T. Peschel, R. Muschall, F. Lederer, S. Trillo, S. Wabinitz, Discrete self-trapping soliton interactions, and beam steering in nonlinear waveguide arrays, Phys. Rev. E 53 (1996) 1172-1189.

[13] H. Eisenberg, R. Morandotti, Y. Silberberg, J. Arnold, G. Pennelli, J. S. Aitchison, Optical discrete solitons in waveguide arrays. 1. soliton formation, J. Opt. Soc. Am. B 19 (2002) 2938-2944.

[14] U. Peschel, R. Morandotti, J. Arnold, J. S. Aitchison, H. Eisenberg, Y. Silberberg, T. Peschel, F. Lederer, Optical discrete solitons in waveguide arrays. 2. dynamics properties, J. Opt. Soc. Am. B 19 (2002) 2637-2644.

[15] S. Jensen, The nonlinear coherent coupler, IEEE J. Quantum Electron. QE18 (1982) 15801583.

[16] S. Trillo, S. Wabinitz, Nonlinear nonreciprocity in a coherent mismatched directional couple, App. Phys. Lett. 49 (1986) 752-754.

[17] D. Christodoulides, R. Joseph, Discrete self-focusing in nonlinear arrays of coupled waveguides, Opt Lett. 13 (1988) 794-796.

[18] T. White, R. McPhedran, C. M. de Sterke, M. Litchinitser, B. Eggleton, Resonance and scattering in microstructured optical fibers, Opt. Lett. 27 (2002) 1977-1979.

[19] T. Pertsch, U. Peschel, J. Kobelke, K. Schuster, H. Bartlet, S. Nolte, A. Tünnermann, F. Lederer, Nonlinearity and disorder in fiber arrays, Phys. Rev. Lett. 93 (2004) 053901.

[20] B. G. Bale, K. Keiu, J. N. Kutz, F. Wise, Transition dynamics for multi-pulsing in modelocked lasers, Opt. Exp. 17 (2009). 
[21] J. M. Soto-Crespo, M. Grapinet, P. Grelu, N. Akhmediev, Bifurcations and multiple-period soliton pulsations in a passively mode-locked fiber laser, Phys. Rev. E 70 (2004).

[22] E. J. Doedel, H. B. Keller, J. P. Kernévez, Numerical analysis and control of bifurcation problems: (ii) bifurcation in infinite dimensions., Int. J. Bifurcation and Chaos 1 (1991) 745-772.

[23] E. J. Doedel, H. B. Keller, J. P. Kernévez, Numerical analysis and control of bifurcation problems: (II) Bifurcation in infinite dimensions., Int. J. Bifurcation and Chaos 1 (1991) 745-772.

[24] W. J. F. Govaerts, Numerical methods for bifurcations of dynamical equilibria, SIAM, Philadelphia, 2000.

[25] H. B. Keller, Numerical methods in bifurcation problems, Springer, New York, 2000.

[26] D. M. Ambrose, J. Wilkening, Computation of time-periodic solutions of the BenjaminOno equation., J. Nonlinear Sci. 20 (2010) 277-308.

[27] D. M. Ambrose, J. Wilkening, Global paths of time-periodic solutions of the BenjaminOno equation connecting pairs of traveling waves., Comm. App. Math. and Comp. Sci. 4 (2009) 177-215.

[28] D. M. Ambrose, J. Wilkening, Computation of symmetric, time-periodic solutions of the vortex sheet with surface tension., Proc. Nat. Acad. Sci. 107 (2010) 3361-3366.

[29] H. B. Keller, Numerical Methods for Two-Point Boundary-Value Problems, Blaisdell, New York, 1968.

[30] J. Stoer, R. Bulirsch, Introduction to Numerical Analysis, Springer, New York, third edition, 2002.

[31] M. O. Bristeau, O. Pironneau, R. Glowinsky, J. Periaux, P. Perrier, On the numerical solution of nonlinear problems in fluid dynamics by least squares and finite element methods. $\mathrm{i}$ - least square formulations and conjugate gradient solution of the continuous problems., Computer Methods in Applied Mechanics and Engineering 17 (1979) 619-657.

[32] A. Jameson, Aerodynamic design via control theory., J. Sci. Comput. 3 (1988) 233-260.

[33] B. Mohammadi, O. Pironneau, Applied Shape Optimization for Fluids, Oxford University Press, New York, 2001.

[34] G. J. Cooper, A. Sayfy, Additive Runge-Kutta methods for stiff ordinary differential equations., Math. Comp. 40 (1983) 207-218.

[35] C. A. Kennedy, M. H. Carpenter, Additive Runge-Kutta schemes for convection-diffusionreaction equations, Appl. Numer. Math. 44 (2003) 139-181.

[36] J. Nocedal, S. J. Wright, Numerical Optimization, Springer, New York, 1999.

[37] C. R. Jones, J. N. Kutz, Stability of mode-locked pulse solutions subject to saturable gain: computing linear stability with the Floquet-Fourier-Hill method, J. Opt. Soc. Am. B (2010) 1184-1194.

[38] S. Wiggins, Introduction to Applied Nonlinear Dynamical Systems and Chaos, Springer, New York, 2nd edition, 1990.

[39] S. Ei, Y. Nishiura, K. Ueda, $2^{n}$ splitting or edge splitting?: a manner of splitting in dissipative systems, Jpn. J. Indust. Appl. Math. 18 (2001) 181-205.

[40] J. R. Dormand, P. J. Prince, A family of embedded Runge-Kutta formulae., J. of Comp. and App. Math. 6 (2006) 19-26.

[41] J. Wilkening, A fifth order additive Runge-Kutta method with a fourth order dense output, Technical Report, Lawrence Berkeley National Laboratory (2010).

[42] U. M. Ascher, S. J. Ruuth, B. T. R. Wetton, Implicit-explicit methods for time-dependent 
partial differential equations, SIAM J. Numer. Anal. 32 (1995) 797-823.

[43] E. Hairer, S. P. Norsett, G. Wanner., Solving Ordinary Differential Equations I: Nonstiff Problems, Springer, Berlin, second edition, 2000.

[44] L. F. Shampine, Some practical Runge-Kutta formulas, Mathematics of Computation 46 (1986) 135-150.

[45] T. Y. Hou, R. Li, Computing nearly singular solutions using pseudo-spectral methods, J. Comput. Phys. 226 (2007) 379-397.

\section{Appendix A. ACM Implementation Flowchart}

In this appendix, we focus on the practical aspects of implementing the Adjoint Continuation Method (ACM) for this problem. In general terms, the ACM operates by treating the task of finding the initial condition of a periodic orbit as an unconstrained minimization problem. One advantage of this approach over, say, the orthogonal collocation method implemented in AUTO [22,23] is that there are many fewer degrees of freedom to compute (as only the initial conditions are unknown). For example, in a typical simulation, we use 1024 grid points in space and 500 timesteps, each broken into 8 Runge-Kutta stages. In the ACM, a fraction of the highest frequency Fourier modes of the initial condition are set to zero, leaving roughly 400 (complex) Fourier modes in each waveguide to be determined by the algorithm. This yields a nonlinear optimization problem in 2400 (real) unknowns. By contrast, to achieve the same accuracy with AUTO, one would need at least 400 (complex) Fourier modes per collocation point. With three waveguides, this yields 2400 (real) unknowns at each collocation point within a timestep. Thus, even if the number of timesteps were reduced to 100 and only 3 collocation points were used per timestep, we would have to solve a nonlinear system of equations with 720,000 unknowns. While it is certainly possible to solve systems of this size, the ACM yields a smaller scale optimization problem that can easily be solved on a laptop computer.

Figure A.15 is a flowchart of the ACM that describes the major steps of the algorithm. In principle, any nonlinear minimization routine could be used to compute the initial conditions of periodic orbits. In our case, we use the BFGS algorithm [36] described in Section 3. In each iteration of the minimization procedure, BFGS supplies an initial condition vector $X(0)=\left(u(0, t), v(0, t), w(0, t), g_{0}, \theta, Z\right)$ for which we must efficiently compute the functional $G$ and its gradient $\nabla_{X} G$ as discussed in Appendix B. Indeed, the majority of the time spent in the ACM will be repeatedly computing these two quantities.

In computing a branch of periodic solutions, several thousand PDE solves are typically required. Therefore, having an efficient time-stepping routine is vital. The main numerical issue with operators such as the one found in this 


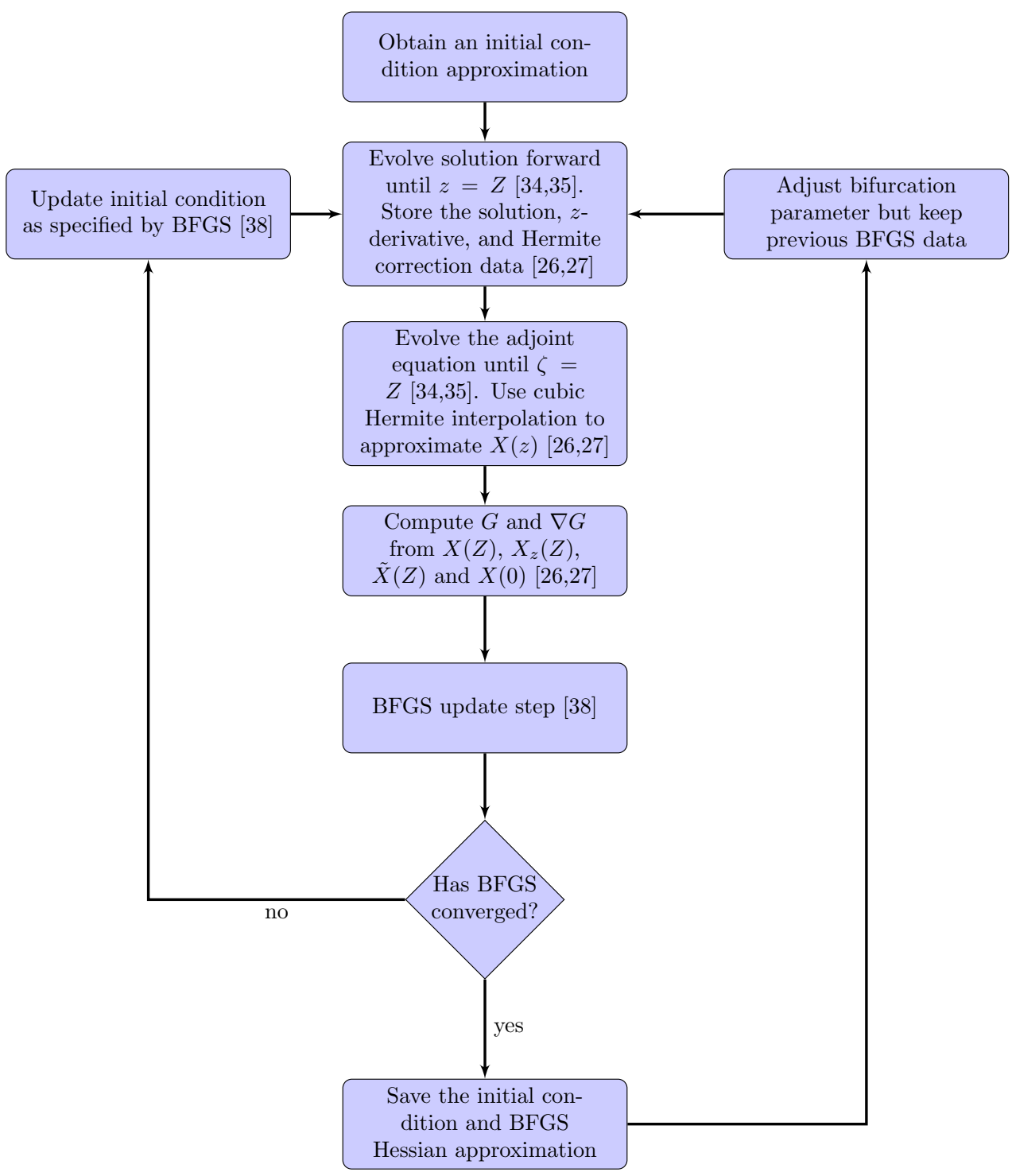

Figure A.15: (Color online, two column) Flowchart for implementing the ACM for the WGAML model. In this chart, $z$ is the time-like parameter, and $X(z)$ is a vector of the degrees of freedom the BFGS algorithm can adjust as they are evolved by the forward equation. $\tilde{X}(\zeta)$ is a vector of those same degrees of freedom as they are evolved by the adjoint equation. Here $\zeta=Z-z$, and $Z$ is the current estimate of the period. Related references are also included.

manuscript is stiffness. Explicit methods like dopri5 require small $\Delta z$ in order 
to remain stable [40]. Fully implicit methods, on the other hand, are too computationally intensive to be a viable replacement. Additive Runge-Kutta (ARK) formulae like the one discussed in Appendix $\mathrm{C}$ and [41] are a good compromise. For the WGAML model, using dopri5 with 1024 Fourier modes in $t$ requires approximately 5000 steps in $z$ to have the same stability and accuracy properties as 500 steps in $z$ with an ARK formula. When using ARK, it is important to treat all the sources of stiffness implicitly. As an example, if the bandwidth limited gain term is not treated implicitly, then 2000 steps in $z$ are required for the ARK method to be stable.

For one particular continuation parameter value, we loop until the BFGS algorithm has converged. At each step, the BFGS algorithm will update the approximation of the Hessian from the function and gradient information it receives until a solution is found. Once the approximation of the Hessian is sufficiently good, the BFGS algorithm can be shown to converge super-linearly. After a single limit cycle is obtained, it is important to preserve the BFGS approximation of the Hessian. If the Hessian is not saved, the subsequent step will still converge, but a large number of the initial iterations will go towards re-estimating the Hessian. By reusing the old Hessian data, fewer iterations are spent on corrections to the Hessian and the quadratic convergence will be reestablished more quickly.

Although finding the solution branches is the most computationally intensive part of the ACM, potentially the most time-consuming part to the user is obtaining a sufficiently good approximation of the periodic solution so that BFGS converges at all. This most commonly occurs when trying to find the branch of periodic solutions created by a Hopf bifurcation or when trying to switch branches due to a bifurcation of the solution branch such as a period-doubling bifurcation. In both cases, it is possible to have accurate information about the bifurcating eigenfunction either by using the Floquet-Fourier-Hill method on stationary solutions [37] or by computing the monodromy matrix [38] for period-one orbits. With either technique, the stationary or period-one solution can be perturbed by the unstable eigenfunction. The most general technique is to temporarily switch the bifurcation parameter to the largest amplitude Fourier mode of the unstable eigenfunction. By switching to this parameter, we are more likely to converge to the new branch of solutions rather than back to the stationary or period-one solution branch. This technique can work even if the resulting branch of solutions is unstable, but success is by no means guaranteed. For problems where the bifurcating branch is stable, it is often easier to adiabatically increase the continuation parameter through the bifurcation point while evolving the system forward in $z$. In that way, an estimate of a solution on the new branch of solutions can be ob- 
tained. This is the easiest method for getting onto a branch of solutions, but it will not work if the resulting branch of solutions is unstable.

In this appendix, we have focused on the practical details of implementing the ACM. However the individual components of the ACM have been studied theoretically in previous works. For instance, details and theoretical considerations of the BFGS algorithm can be found in Ref. [36], and Refs. [34, 35] contain additional details about the ARK formulae. Although they are not mentioned in detail here, the theoretical aspects of the ACM have not been neglected. Ultimately, many of the practical benefits discussed in this appendix, such as using a semi-implicit time stepper and reusing the BFGS Hessian, can be understood and explained in terms of the properties of the individual components of the ACM.

\section{Appendix B. The Adjoint PDE to the WGAML}

The goal of the adjoint PDE is to find the variational derivative $\frac{\delta G}{\delta q_{0}}=\left(\frac{\delta G}{\delta u_{0}}, \frac{\delta G}{\delta v_{0}}, \frac{\delta G}{\delta w_{0}}, \frac{\partial G}{\partial g_{0}}\right) \in$ $X$, which satisfies

$$
\left.\frac{d}{d \varepsilon}\right|_{\varepsilon=0} G\left(q_{0}+\varepsilon \dot{q}_{0}, Z, \theta\right)=\left\langle\frac{\delta G}{\delta q_{0}}, \dot{q}_{0}\right\rangle
$$

for every sufficiently smooth $\dot{q}_{0} \in X$. Here a dot represents a directional derivative with respect to the initial conditions, and we will write $\dot{G}$ for the left hand side of (B.1). The quantities in (6) are the real and imaginary parts of the Fourier coefficients of $\frac{\delta G}{\delta u_{0}}, \frac{\delta G}{\delta v_{0}}$ and $\frac{\delta G}{\delta w_{0}}$, e.g.

$$
\frac{\partial G}{\partial \mathfrak{R}\left(\hat{u}_{k}\right)}=\left\langle\frac{\delta G}{\delta q_{0}},\left(\begin{array}{c}
e^{i k t} \\
0 \\
0 \\
0
\end{array}\right)\right\rangle=2 \pi \mathfrak{R}\left\{\left(\frac{\delta G}{\delta u_{0}}\right)_{k}\right\}, \quad \frac{\partial G}{\partial \mathfrak{J}\left(\hat{u}_{k}\right)}=\left\langle\frac{\delta G}{\delta q_{0}},\left(\begin{array}{c}
i e^{i k t} \\
0 \\
0 \\
0
\end{array}\right)\right\rangle=2 \pi \mathfrak{I}\left\{\left(\frac{\delta G}{\delta u_{0}}\right)_{k}\right\} .
$$

To find a formula for $\frac{\delta G}{\delta q_{0}}$, we evaluate the left hand side of (B.1) and then manipulate the result to obtain the form on the right of (B.1). On the left, we have

$$
\dot{G}:=\left.\frac{d}{d \varepsilon}\right|_{\varepsilon=0} G\left(q_{0}+\varepsilon \dot{q}_{0}, Z, \theta\right)=\left\langle q(Z, \cdot)-q_{0}(\cdot), \dot{q}(Z, \cdot)-\dot{q}_{0}(\cdot)\right\rangle
$$


where $\dot{q}(z, t)$ solves the linearization of $(1 \mathrm{~d})-(1 \mathrm{~g})$ about $q(z, t)$ with initial condition $\dot{q}(0, t)=\dot{q}_{0}(t)$ :

$$
\begin{aligned}
& i \frac{\partial \dot{u}}{\partial z}+\frac{D}{2} \frac{\partial^{2} \dot{u}}{\partial t^{2}}+2 \beta|u|^{2} \dot{u}+\beta u^{2} \dot{u}^{*}+C \dot{v}+i \gamma_{0} \dot{u}-\frac{2 i g_{0}}{1+\|u\|^{2} / e_{0}}\left(1+\tau \frac{\partial^{2}}{\partial t^{2}}\right) \dot{u} \\
& +\left[\frac{4 i g_{0}\langle u, \dot{u}\rangle / e_{0}}{\left(1+\|u\|^{2} / e_{0}\right)^{2}}-\frac{2 i \dot{g}_{0}}{1+\|u\|^{2} / e_{0}}\right]\left[\left(1+\tau \frac{\partial^{2}}{\partial t^{2}}\right) u\right]=\theta \dot{u} \\
& i \frac{\partial \dot{v}}{\partial z}+C(\dot{w}+\dot{u})+i \gamma_{1} \dot{v}=\theta \dot{v} \\
& i \frac{\partial \dot{w}}{\partial z}+C \dot{v}+i \gamma_{2} \dot{w}=\theta \dot{w} \\
& \frac{\partial \dot{g}_{0}}{\partial z}=0 .
\end{aligned}
$$

Here $\langle u, \dot{u}\rangle=\left(\int_{-\pi}^{\pi} \mathfrak{R}\left\{u(z, t)^{*} \dot{u}(z, t)\right\} d t\right)$ is a real number, and depends on $z$. Next we define adjoint variables $\tilde{q}(\zeta, t)=\left(\tilde{u}(\zeta, t), \tilde{v}(\zeta, t), \tilde{w}(\zeta, t), \tilde{g}_{0}\right)$, where $\zeta=Z-z$ is a reversed time-like variable. We define $\tilde{q}_{0}(t)=q(Z, t)-q_{0}(t)$ so that (B.2) becomes

$$
\dot{G}=\left\langle\tilde{q}_{0}(\cdot), \dot{q}(Z, \cdot)\right\rangle-\left\langle\tilde{q}_{0}, \dot{q}_{0}\right\rangle .
$$

This can be put in the desired form (B.1) with

$$
\frac{\delta G}{\delta q_{0}}(t)=\tilde{q}(Z, t)-\tilde{q}_{0}(t)
$$

provided that $\langle\tilde{q}(Z-z, \cdot), \dot{q}(z, \cdot)\rangle$ remains constant for $0 \leq z \leq Z$. Differentiation with respect to $z$ shows that this condition will hold provided that $\tilde{q}(\zeta, t)$ satisfies

$$
\left\langle\frac{\partial \tilde{q}}{\partial \zeta}(\zeta, \cdot), \dot{q}(z, \cdot)\right\rangle=\left\langle\tilde{q}(\zeta, \cdot), \frac{\partial \dot{q}}{\partial z}(z, \cdot)\right\rangle, \quad \tilde{q}(0, t)=\tilde{q}_{0}(t), \quad \zeta=Z-z
$$


Using (B.3) to evaluate $\left\langle\tilde{q}(\zeta, \cdot), \frac{\partial \dot{q}}{\partial z}(z, \cdot)\right\rangle$, we integrate by parts and collect terms to identify the adjoint system

$$
\begin{aligned}
\frac{\partial \tilde{u}}{\partial \zeta}=-i \frac{D}{2} & \frac{\partial^{2} \tilde{u}}{\partial t^{2}}-2 i \beta|u|^{2} \tilde{u}+i \beta u^{2} \tilde{u}^{*}-\gamma_{0} \tilde{u} \\
& \quad+\frac{2 g_{0}}{1+\|u\|^{2} / e_{0}}\left(1+\tau \frac{\partial^{2}}{\partial t^{2}}\right) \tilde{u}-\frac{4 g_{0}\left\langle\tilde{u},\left(1+\tau \partial_{t}^{2}\right) u\right\rangle}{e_{0}\left(1+\|u\|^{2} / e_{0}\right)^{2}} u+i \theta \tilde{u}-i C \tilde{v}
\end{aligned}
$$

In this equation, as $\zeta$ runs from 0 to $Z$, the variables $u, v$ and $w$ are evaluated at $(Z-\zeta, t)$ while $\tilde{u}, \tilde{v}$ and $\tilde{w}$ are evaluated at $(\zeta, t)$. Then using (B.1) and (B.4) in combination with (B.5) the remaining components of the gradient can be computed.

\section{Appendix C. Semi-implicit Runge-Kutta Method}

The terms involving second derivatives with respect to the space-like variable, $t$, cause the systems (1) and (B.5) to be stiff. This means stability requires smaller steps in an explicit scheme than is necessary for accuracy. On the other hand, a fully implicit method would be difficult to implement due to the fact that (1) is nonlinear and (B.5), while linear, is not diagonalized by the Fourier transform. The idea of an implicit-explicit (IMEX) multi-step method [42], or an additive Runge-Kutta (ARK) method [34, 35], is to write the evolution equation as a sum

$$
\frac{\partial q}{\partial z}=f(z, q)+g(z, q)
$$

and devise a scheme in which $f$ is treated explicitly, $g$ is treated implicitly, the scheme for $g$ alone is e.g. $L$-stable, and the combined scheme is high order. In the ARK framework, there are two sets of stage derivatives and two Butcher arrays 
[43], one for $f$ and one for $g$ :

$$
\begin{aligned}
& \begin{array}{ll}
k_{i}=f\left(z_{n}+c_{i} h, q_{n}+h \sum_{j} a_{i j} k_{j}+h \sum_{j} \hat{a}_{i j} \ell_{j}\right), \\
\ell_{i}=g\left(z_{n}+\hat{c}_{i} h, q_{n}+h \sum_{j} a_{i j} k_{j}+h \sum_{j} \hat{a}_{i j} \ell_{j}\right),
\end{array} \quad \begin{array}{l|l}
b^{T} \\
b^{T}
\end{array} \quad \begin{array}{ll}
\hat{c} & \hat{A} \\
b^{T}
\end{array} \\
& q_{n+1}=q_{n}+h \sum_{j} b_{j} k_{j}+h \sum_{j} \hat{b}_{j} \ell_{j} . \quad \text { for } f \quad \text { for } g
\end{aligned}
$$

The Butcher arrays satisfy $a_{i j}=0$ if $i \leq j$ and $\hat{a}_{i j}=0$ if $i<j$. This allows the stage derivatives to be solved for in order, $l_{1}, k_{1}, \ldots, l_{s}, k_{s}$, where $s$ is the number of stages of the scheme. We used a variant [41] of the 5th order ARK scheme of Kennedy and Carpenter [35], modified so that a fourth order dense output exists. A dense output [43] is a rule for accurately interpolating the solution between timesteps. In our case, we must interpolate the solution of the forward problem (1) to solve the adjoint problem. To achieve fifth order accuracy in the adjoint system, we require a fourth order dense output formula. We use the dense output to generate quartic corrections [44] to cubic Hermite interpolation. Details are given in [41].

For the nonlinear system (1d)-(1f), we set $q=(u, v, w)$ in (C.1) and define

$$
\begin{aligned}
& f(q)=\left(\begin{array}{ccc}
-i \theta+i \beta|u|^{2}-\gamma_{0}+\frac{2 g_{0}}{1+\|u\|^{2} / e_{0}} & i C & 0 \\
i C & -i \theta-\gamma_{1} & i C \\
0 & i C & -i \theta-\gamma_{2}
\end{array}\right)\left(\begin{array}{l}
u \\
v \\
w
\end{array}\right), \\
& g(q)=\left(\begin{array}{ccc}
\left(i \frac{D}{2}+\frac{2 g_{0} \tau}{1+\|u\|^{2} / e_{0}}\right) \frac{\partial^{2}}{\partial t^{2}} & 0 & 0 \\
0 & 0 & 0 \\
0 & 0 & 0
\end{array}\right)\left(\begin{array}{c}
u \\
v \\
w
\end{array}\right) .
\end{aligned}
$$

Note that there is no need to include $(1 \mathrm{~g})$ in the evolution equations once the adjoint system is derived; $g_{0}$ is simply treated as a parameter in (C.1) and (C.3). The main challenge in this procedure is that $g(q)$ in $(\mathrm{C} .3)$ is non-linear due to the term $\|u\|^{2}$. The implicit equation that must be solved has the form

$$
(U, V, W)=g[(u, v, w)+\varepsilon(U, V, W)],
$$

where $(U, V, W)=l_{i}$ in (C.2), $\varepsilon=\hat{a}_{i i} h$, and $(u, v, w)=q_{n}+h \sum_{j=1}^{i-1}\left[a_{i j} k_{j}+\hat{a}_{i j} l_{j}\right]$. Due to the form of $g(q)$ in (C.3), we have $V=W=0$. We solve for $U$ in Fourier space:

$$
\hat{U}_{k}+k^{2}\left(i \frac{D}{2}+\frac{2 g_{0} \tau}{\left.1+\left(2 \pi / e_{0}\right) \sum_{j} \mid \hat{u}_{j}+\varepsilon \hat{U}_{36}\right)^{2}}\right)\left(\hat{u}_{k}+\varepsilon \hat{U}_{k}\right)=0, \quad(k \in \mathcal{K}) .
$$


Here $\mathcal{K}=\{-N / 2+1, \ldots, N / 2-1\}$, where $N$ is the number of Fourier collocation points used to discretize $(-\pi, \pi]$, and we set the Nyquist mode to zero, $\hat{U}_{N / 2}=0$. In addition, at the end of each time-step, we apply a 36th order filter [45] in which the Fourier modes are multiplied by $e^{-36(2|k| / N)^{36}}$. This filter has proved successful in suppressing aliasing errors while allowing high frequency modes to contribute useful information to the solution.

It is convenient to solve for the real and imaginary parts of $\hat{U}_{k}$ in (C.5) separately. Setting

$$
\hat{U}_{k}=a_{k}+i b_{k}, \quad \hat{u}_{k}=\alpha_{k}+i \beta_{k},
$$

the system (C.5) becomes

$$
F_{k}=\left(\begin{array}{l}
a_{k} \\
b_{k}
\end{array}\right)+k^{2}\left(\frac{D}{2} J+E(r) I\right)\left(\begin{array}{c}
\alpha_{k}+\varepsilon a_{k} \\
\beta_{k}+\varepsilon b_{k}
\end{array}\right)=0, \quad(k \in \mathcal{K}),
$$

where

$E(r)=\frac{2 g_{0} \tau}{1+\left(2 \pi / e_{0}\right) r}, \quad r=\sum_{j}\left[\left(\alpha_{j}+\varepsilon a_{j}\right)^{2}+\left(\beta_{j}+\varepsilon b_{j}\right)^{2}\right], \quad I=\left(\begin{array}{cc}1 & \\ & 1\end{array}\right), \quad J=\left(\begin{array}{cc}0 & -1 \\ 1 & 0\end{array}\right)$.

We solve (C.6) iteratively, using Newton's method

$$
\hat{U}^{(v+1)}=\hat{U}^{(v)}-\left[\nabla F\left(\hat{U}^{(v)}\right)\right]^{-1} F\left(\hat{U}^{(v)}\right),
$$

where $\hat{U}=\left(\ldots, a_{-1}, b_{-1}, a_{0}, b_{0}, a_{1}, b_{1}, \ldots\right) \in \mathbb{R}^{2 N-2}$. As a starting guess, $\hat{U}^{(0)}$, we drop $\varepsilon a_{j}$ and $\varepsilon b_{j}$ in the formula for $r$ and solve (C.6), which becomes a decoupled set of $2 \times 2$ linear systems when $r$ is frozen in this way. On subsequent iterations of Newton's method, we note that the Jacobian is a rank one perturbation of a $2 \times 2$ block-diagonal matrix:

$(\nabla F)_{k j}=\frac{\partial F_{k}}{\partial\left(a_{j}, b_{j}\right)}=A_{k j}+B_{k j}$

$$
\begin{aligned}
A_{k j} & =\left\{I+\varepsilon k^{2}[(D / 2) J+E(r) I]\right\} \delta_{k j}, \\
B_{k j} & =2 \varepsilon k^{2} E^{\prime}(r)\left(\begin{array}{c}
\alpha_{k}+\varepsilon a_{k} \\
\beta_{k}+\varepsilon b_{k}
\end{array}\right)\left(\alpha_{j}+\varepsilon a_{j}, \beta_{j}+\varepsilon b_{j}\right) .
\end{aligned}
$$

Thus, we may use the Sherman-Morrison formula [36] to invert $\nabla F$ :

$$
\left(A+u v^{T}\right)^{-1}=A^{-1}-\frac{A^{-1} u v^{T} A^{-1}}{1+v^{T} A^{-1} u} .
$$

To invert the $2 \times 2$ diagonal blocks of $A$, we use $(x I+y J)^{-1}=\frac{1}{x^{2}+y^{2}}(x I-y J)$. Because $\varepsilon$ is small in practice, the starting guess is close enough that Newton's method converges to machine precision in 3-5 iterations. 
For the adjoint system (B.5), we use the same fifth order additive RungeKutta scheme as in the forward problem (1d)-(1f), but now the equations are non-autonomous and linear

$$
\frac{\partial \tilde{q}}{\partial \zeta}=f(\zeta, \tilde{q})+g(\zeta, \tilde{q}), \quad \tilde{q}=\left(\tilde{u}, \tilde{v}, \tilde{w}, \tilde{g}_{0}\right) .
$$

We set

$$
g(\zeta, \tilde{q})=\left(\begin{array}{c}
\left(-i \frac{D}{2}+\frac{2 g_{0} \tau}{1+\|u(Z-\zeta, \cdot)\|^{2} / e_{0}}\right) \frac{\partial^{2} \tilde{u}}{\partial t^{2}} \\
0 \\
0 \\
0
\end{array}\right)
$$

and define $f(\zeta, \tilde{q})$ to be the remaining terms of (B.5). Although $f(\zeta, \tilde{q})$ and $g(\zeta, \tilde{q})$ are both linear functions of $\tilde{q}, f(\zeta, \tilde{q})$ contains terms that are difficult to invert in a fully implicit scheme while $g(\zeta, \tilde{q})$ is easily inverted using the FFT in a similar way to the forward problem above, but without the need for Newton's method. As mentioned at the beginning of this section, we use a dense output formula described in [41] to interpolate the solution $q(z, t)$ between timesteps to evaluate quantities such as $\|u(Z-\zeta, \cdot)\|$ in the adjoint problem. 